\title{
VANISHING COHOMOLOGY FOR HOLOMORPHIC VECTOR BUNDLES IN A BANACH SETTING*
}

\author{
LÁSZLÓ LEMPERT ${ }^{\dagger}$
}

To Yum-Tong Siu, on his sixtieth birthday

\begin{abstract}
For a large class of Banach spaces $X$ we prove the following. If $\Omega \subset X$ is open and pseudoconvex, and $E \rightarrow \Omega$ is a locally trivial holomorphic Banach bundle, then the sheaf cohomology groups $H^{q}(\Omega, E)$ vanish for $q \geq 1$. We also give an application concerning neighborhoods of complex submanifolds.
\end{abstract}

1. Introduction. Consider a complex Banach space $X$, a pseudoconvex open $\Omega \subset X$, a holomorphic Banach bundle $E \rightarrow \Omega$ (that is, a holomorphic vector bundle with fibers isomorphic to Banach spaces), and the sheaf cohomology groups $H^{q}(\Omega, E)$. The problem we shall address here is whether these groups vanish for $q \geq 1$. What is customarily called Cartan's Theorem B implies that this is so if $\operatorname{dim} X<\infty$; Cartan himself dealt with bundles of finite rank and Leiterer extended his result to Banach bundles, see $[\mathrm{C}, \mathrm{Li}]$. It is possible that $H^{q}(\Omega, E)=0, q \geq 1$, holds in complete generality, but at present, and to this author, such a theorem is very much out of reach. Instead, as in earlier vanishing theorems in [Lm2, P1-3], we restrict ourselves to Banach spaces with bases.

Recall that $X$ has a Schauder basis $e_{1}, e_{2}, \ldots \in X$ if any $x \in X$ can be uniquely represented as a norm convergent sum

$$
x=\sum_{n=1}^{\infty} \lambda_{n} e_{n}, \quad \lambda_{n} \in \mathbb{C} .
$$

If in addition the series in (1.1) converge after arbitrary rearrangements, one speaks of a countable unconditional basis. The spaces $l^{p}, L^{p}[0,1]$ for $1<p<\infty, l^{1}$, and the space $c$ of convergent sequences all have unconditional bases, see [Sn]. Our main result is

THEOREM 1.1. If $X$ is a Banach space with countable unconditional basis, $\Omega \subset X$ open and pseudoconvex, $E \rightarrow \Omega$ a locally trivial holomorphic Banach bundle, and $q \geq 1$, then $H^{q}(\Omega, E)=0$.

This theorem will be derived from a variant, in which $X$ is only required to have a Schauder basis and a property that involves Runge approximation. Let $B(x, r) \subset X$ denote the ball of radius $r$, centered at $x$, and $B(0, r)=B(r)$. If $M$ is a complex manifold and $E \rightarrow M$ a holomorphic fiber bundle, $\mathcal{O}(M, E)$ stands for the space of holomorphic sections; when $E=M \times W$ is trivial, we shall write $\mathcal{O}(M ; W)$ for $\mathcal{O}(M, E)$. Consider the following

\footnotetext{
* Received April 9, 2003; accepted for publication May 28, 2003.

† Department of Mathematics, Purdue University, West Lafayette, IN 47907-1395, USA. (lempert@math.purdue.edu). This paper was written while, on leave from Purdue University, I was enjoying the hospitality of the Analysis Department, Eötvös University, Budapest. I am grateful to both institutions. Partial support from the NSF is also gratefully acknowledged.
} 
Hypothesis 1.2. There is a $\mu \in(0,1)$ such that for any Banach space $\left(W,\|\|_{W}\right)$, $\varepsilon>0$, and $g \in \mathcal{O}(B(1) ; W)$ an $h \in \mathcal{O}(X ; W)$ can be found that satisfies $\|g-h\|_{W}<\varepsilon$ on $B(\mu)$.

In [Lm3] we proved Hypothesis 1.2 for $X$ that has a countable unconditional basis; Josefson gave a different proof in $[\mathrm{J}]$. Therefore Theorem 1.1 follows from

TheOREM 1.3. Suppose $X$ is a Banach space with a Schauder basis and Hypothesis 1.2 holds. If $\Omega \subset X$ is open and pseudoconvex, $E \rightarrow \Omega$ a locally trivial holomorphic Banach bundle, and $q \geq 1$, then $H^{q}(\Omega, E)=0$.

We shall also prove an infinite dimensional extension of Cartan's Theorem A to the effect that holomorphic sections of $E$ over certain subsets of $\Omega$ can be approximated by global sections, see Theorem 4.5. Further, we shall apply Theorem 1.3 to study neighborhoods of submanifolds, in the spirit of Docquier-Grauert and Siu [DG, Su]. We say that a complex submanifold $M$ of a complex manifold $M^{\prime}$ is split if $T_{x} M \subset$ $T_{x} M^{\prime}$ has a closed complement for each $x \in M$.

TheOREM 1.4. Suppose $X$ is a Banach space with a Schauder basis, Hypothesis 1.2 holds, $O \subset X$ is open, and $M \subset O$ is a split closed complex submanifold that is biholomorphic to a pseudoconvex open subset of a Banach space. Identify $M$ with the image of the zero section in the normal bundle $\bar{\nu}=(T X \mid M) / T M$. Then a neighborhood of $M$ in $\bar{\nu}$ admits a biholomorphism on a pseudoconvex neighborhood of $M$ in $O$. On $M$ the biholomorphism can be chosen to be the identity.

In a related development, Patyi proved that subvarieties $M$ of pseudoconvex open $\Omega \subset X$ have pseudoconvex neighborhood bases, provided $M$ can be defined as the zero locus of a holomorphic section of a Banach bundle $E \rightarrow \Omega$, see [P3, Theorem 1.2].

Next comes a simplified and therefore not completely accurate discussion of the proof of Theorem 1.3. A general strategy to prove cohomology vanishing, in finite or infinite dimensions, is to exhaust $\Omega$ by a sequence of simpler sets $\Omega_{N} \subset \Omega, N=1,2, \ldots$. so that

$1^{\circ} H^{q}(\Omega, E) \mid \Omega_{N}=0$ can be proved, and

$2^{\circ}$ holomorphic sections of $E$ over (certain subsets of) $\Omega_{N}$ can be approximated by global holomorphic sections.

The approximation property can then be used to show that

$$
H^{q}(\Omega, E)=\lim _{N} H^{q}(\Omega, E) \mid \Omega_{N}=0 .
$$

When $\operatorname{dim} \Omega<\infty$, Cartan and his followers take $\Omega_{N}$ compact and holomorphically convex. Over a compact $\Omega_{N}$ any cohomology class $f \in H^{q}(\Omega, E)$ is represented by a Čech cocyle of a finite covering, which allows for an inductive argument to prove $f \mid \Omega_{N}=0$. By contrast, in infinite dimensions $\Omega$ can not be exhausted by a sequence of compacta.

Instead, in [Lm2] we exhausted an infinite dimensional $\Omega$ by pseudoconvex open subsets; each $\Omega_{N}$ was a ball bundle over a finite dimensional base $D_{N}$. For trivial $E$ we could prove $1^{\circ}$ and $2^{\circ}$, and ultimately $H^{q}(\Omega, E)=0$. The case of a general bundle was subsequently taken up by Patyi. In $[\mathrm{P} 1, \mathrm{P} 2]$ he used the same $\Omega_{N}$ and was able to show $1^{\circ}$ and $2^{\circ}$, provided $E \mid \Omega_{N}$ is isomorphic to the bundle induced from $E \mid D_{N}$ by the projection $\Omega_{N} \rightarrow D_{N}$; if this is so, Patyi calls $E \mid \Omega_{N}$ a basic bundle. (There is a subtlety in the proof and even in the formulation of $2^{\circ}$. Above we have not 
specified in what topology the approximation is to be done. When $\operatorname{dim} \Omega<\infty$ and the $\Omega_{N}$ are compact, the approximation is uniform on $\Omega_{N}$. When $\operatorname{dim} \Omega=\infty$ and $E$ is trivial, the approximation is still uniform, although only on certain subsets $\omega_{N} \subset \Omega_{N}$; but for general $E$ uniform approximation over noncompact sets is meaningless. Patyi had to construct appropriate fiber metrics on $E \mid \Omega_{N}$, in which he was then able to approximate uniformly, as needed.) Furthermore, he devised an approach to prove $E \mid \Omega_{N}$ is always basic. This depended on a Runge type approximation hypothesis for Lie group valued holomorphic functions. He could verify the hypothesis for solvable groups, so that in the end he proved $H^{q}(\Omega, E)=0$ for $E$ with solvable structure group. However, it is still unknown whether his approximation hypothesis is generally true.

In this paper we improve on Patyi's scheme in the following way. Let $Z$ be the typical fiber of $E$. It is easy to find an open covering $\mathfrak{U}$ of $\Omega$ and holomorphic trivializations $e_{U}$ of $E \mid U, U \in \mathfrak{U}$, such that the transition maps $e_{U} e_{V}^{-1}: U \cap V \rightarrow$ $\mathrm{GL}(Z)$ are bounded and uniformly continuous. We shall call the choice of $\left\{e_{U}\right\}_{U \in \mathfrak{U}}$ a uniformization of $E$, and if for a given covering $\mathfrak{U}$ a uniformization exists, we shall say $E$ is $\mathfrak{U}$-uniform. Next we exhaust $\Omega$ by pseudoconvex open $\Omega_{N}$, each $\Omega_{N}$ a ball bundle over a finite dimensional base $D_{N}$, in a way adapted to the uniformization. Namely, $\mathfrak{U} \mid \Omega_{N}$ will have a finite refinement $\mathfrak{U}_{N}$ that is induced from a covering of the base $D_{N}$. Thus $E \mid \Omega_{N}$ is $\mathfrak{U}_{N}$-uniform, and we shall show that such bundles are basic. This could be done following Patyi's idea, because all one needs now is to approximate bounded holomorphic functions with values in $\mathrm{GL}(Z)$, which is easy to do. However, we shall proceed differently, bypass approximation, and reduce the problem to finite dimensions instead. Once $E \mid \Omega_{N}$ is known to be basic, $1^{\circ}$ and $2^{\circ}$ can be proved as in $[\mathrm{P} 1]$.

The novelty in our approach is the concept of uniformization and the construction of adapted $\Omega_{N}$. This latter is based on the following result, cf. [Lm4, Theorem 1.6].

PluRisubHARMONiC DOMINATION. If a Banach space $X$ has a Schauder basis and satisfies Hypothesis 1.2, $\Omega \subset X$ is open and pseudoconvex, and $u: \Omega \rightarrow \mathbb{R}$ is locally bounded above, then there is a plurisubharmonic $v: \Omega \rightarrow \mathbb{R}$ such that $u \leq v$.

Here is a brief overview of the contents. In the preparatory Section 2 we discuss boundedness and uniform continuity of functions with values in Banach-Lie groups. The results obtained will be used in Section 3, that introduces the idea of uniformization both for vector and principal bundles. For suitably uniformized Banach bundles over certain ball bundles we prove a version of Theorems A and B, Corollary 3.5. In Section 4 general uniformized Banach bundles $E \rightarrow \Omega$ are considered, and we exhaust $\Omega$ by $\Omega_{N}$ to which the results of Section 3 apply. The main results here, Theorem 4.5 and Proposition 4.6 concern approximation. Section 5 ties together the loose ends, and proves Theorem 1.3. Theorem 1.4 is proved in Section 6 .

For background and basic complex analysis/geometry in finite and infinite dimensions the reader is referred to $[\mathrm{H}, \mathrm{Lm} 1, \mathrm{M}, \mathrm{N}]$.

2. Calculus of group valued functions. In this section we shall define and discuss bounded and uniformly continuous functions with values in a Banach-Lie group. Fix a smooth real Banach-Lie group $G$.

Definition 2.1. A subset $S \subset G$ is bounded if for an arbitrary neighborhood $U$ of $1 \in G$ there is a finite set $F \subset G$ and $n \in \mathbb{N}$ such that $S \subset F U^{n}$.

When $S$ is a bounded subset of the identity component $G_{1}$ of $G$, for any neighbor- 
hood $U$ of 1 there will be a $k \in \mathbb{N}$ such that $S \subset U^{k}$. It will suffice to check this when $U=U^{-1} \subset G_{1}$. The subgroup generated by $U$ is $G_{1}$, since it is open, hence closed. It follows that any finite $E \subset G_{1}$ is contained in some $U^{m}$. Therefore, if $S \subset F U^{n}$ with a finite $F \subset G$ then $S \subset\left(F \cap G_{1}\right) U^{n} \subset U^{n+m}$, as claimed.

Let $\mathfrak{g}$ be the (Banach-) Lie algebra of $G$ and exp: $\mathfrak{g} \rightarrow G$ the exponential map, a diffeomorphism near 0 . Since for a bounded neighborhood $\mathfrak{u}$ of $0 \in \mathfrak{g}$ the sets $\mathfrak{u} / j, j \in \mathbb{N}$, form a neighborhood basis of $0, \exp (\mathfrak{u} / j)$ form a neighborhood basis of $1 \in G$. Since furthermore $\exp \mathfrak{u} \subset(\exp \mathfrak{u} / j)^{j}$, it follows that $U=\exp \mathfrak{u}$ is bounded, and even $U^{k}$ is bounded for each $k \in \mathbb{N}$.

Proposition 2.2. If $S, T \subset G$ are bounded, so are $S T$ and $S^{-1}$.

Proof. Boundedness of $S T$ follows from [A, (3.2) Lemma]. As to $S^{-1}$, take a symmetric neighborhood $U$ of 1 so that $U^{k}$ is bounded for any $k \in \mathbb{N}$, as above. There are a finite set $F \subset G$ and $n \in \mathbb{N}$ such that $S \subset F U^{n}$. Then $S^{-1} \subset U^{-n} F^{-1}=U^{n} F^{-1}$ is bounded by the first part of the proposition.

Now fix another smooth Banach-Lie group $H$, let $S \subset H$ and $f: S \rightarrow G$.

Definition 2.3. (a) $f$ is bounded if $f(S) \subset G$ is bounded.

(b) $f$ is uniformly continuous if for every neighborhood $U \subset G$ of $1_{G}$ there is a neighborhood $V \subset H$ of $1_{H}$ such that $f(a)^{-1} f(b) \in U$ whenever $a^{-1} b \in V, a, b \in S$.

In verifying uniform continuity we shall depend on the following

Lemma 2.4. Let $A, B, C$ be Banach manifolds, $b_{0} \in B, c_{0} \in C$, and $F: A \times B \rightarrow$ $C$ a smooth map such that $F\left(\cdot, b_{0}\right) \equiv c_{0}$. Any $a_{0} \in A$ has a neighborhood $A_{0}$ with the following property: given a neighborhood $U$ of $c_{0}$ there is a neighborhood $B_{U}$ of $b_{0}$ with $F\left(A_{0} \times B_{U}\right) \subset U$.

Proof. It can be assumed that $A, B, C$ are convex open subsets of Banach spaces and $a_{0}, b_{0}, c_{0}$ are at the origin. Then

$$
F(a, b)=\int_{0}^{1} \frac{d}{d t} F(a, t b) d t=\left(\int_{0}^{1}\left(\partial_{B} F\right)(a, t b) d t\right) b,
$$

$\partial_{B}$ denoting partial differential along $B$. If $A_{0} \subset A, B_{0} \subset B$ are convex neighborhoods of the origin such that the linear maps $\partial_{B} F(a, b)$ are uniformly bounded as $a, b$ range over $A_{0}, B_{0}$, then the integral on the right of (2.1) will be uniformly bounded, and the claim follows (with some $B_{U} \subset B_{0}$ ).

Corollary 2.5. If $S \subset H$ is open and $f: S \rightarrow G$ is smooth then each point of $S$ has a neighborhood on which $f$ is bounded and uniformly continuous.

Proof. Local boundedness directly follows from the mere continuity of $f$. As for local uniform continuity, given $a_{0} \in S$, choose neighborhoods $A$ of $a_{0}$ and $B$ of $1_{H}$ so that $A B \subset S$; let $b_{0}=1_{H}, C=G, c_{0}=1_{G}$, and $F(a, b)=f(a)^{-1} f(a b)$. Lemma 2.4 then produces a neighborhood $A_{0}$ of $a_{0}$ on which $f$ is uniformly continuous.

Now consider the adjoint map

$$
\operatorname{Ad}_{g}: G \ni x \mapsto g^{-1} x g \in G, \quad g \in G .
$$


Corollary 2.6. If $S \subset G$ is bounded then for any neighborhood $U$ of $1 \in G$ there is another neighborhood $V$ of 1 with $\operatorname{Ad}_{S} V \subset U$.

Proof. Setting $A, B, C=G, b_{0}=1$, and $F(a, b)=\operatorname{Ad}_{a} b$ in Lemma 2.4 we obtain a neighborhood $W$ of $a_{0}=1$ so that the claim holds for $S=W$. Since it also holds for finite $S$, and if it holds for $S$ and $T$ it will hold for $S T$-indeed $\operatorname{Ad}_{S T} V \subset\left(\operatorname{Ad}_{S} V\right)\left(\operatorname{Ad}_{T} V\right)$-, the corollary follows.

TheOREM 2.7. (a) Multiplication $G \times G \rightarrow G$ is bounded and uniformly continuous on bounded sets.

(b) Inversion $G \rightarrow G$ and exp: $\mathfrak{g} \rightarrow G$ are bounded and uniformly continuous on bounded sets.

(c) $0 \in \mathfrak{g}$ has a bounded neighborhood $\mathfrak{u}_{0}$ such that $\exp \mid \mathfrak{u}_{0}$ has a bounded and uniformly continuous inverse on $U_{0}=\exp \mathfrak{u}_{0}$, denoted $\log$.

Proof. (a) follows from Proposition 2.2, the identity $(a b)^{-1}(c d)=\left(b^{-1} d\right) \operatorname{Ad}_{d}\left(a^{-1} c\right)$, and Corollary 2.6. To take care of inversion one has to use the identity $\left(a^{-1}\right)^{-1} b^{-1}=$ $\operatorname{Ad}_{a^{-1}}\left(b^{-1} a\right)$ instead. As to the exponential map, we already know it maps bounded sets to bounded sets. Corollary 2.5 implies $0 \in \mathfrak{g}$ has a convex, bounded neighborhood on which exp is uniformly continuous. If $\mathfrak{u}$ is such a neighborhood then so is $2 \mathfrak{u}$ by the identity

$$
(\exp 2 a)^{-1} \exp 2 b=(\exp a)^{-1}(\exp b) \operatorname{Ad}_{\exp b}(\exp a)^{-1} \exp b
$$

and Corollary 2.6; hence by induction exp is indeed uniformly continuous on all bounded sets. Finally, (c) follows from Corollary 2.5, since log is smooth on some neighborhood of $1 \in G$.

TheOREM 2.8. (a) A homomorphism $\varphi: H \rightarrow G$ is uniformly continuous. It is also bounded on bounded sets.

(b) A smooth action $\psi: H \times G \rightarrow G$ of $H$ by automorphisms of $G$ is bounded and uniformly continuous on bounded sets.

Proof. (a) Uniform continuity is obvious. As $\varphi$ is bounded on some neighborhood of 1 , Proposition 2.2 implies the rest.

(b) Write $\psi(h, g)=\psi_{h}(g)$. To prove boundedness, first take a connected neighborhood $W \times U$ of $1_{H \times G}$ on which $\psi$ is bounded. As $\psi\left(W \times U^{n}\right) \subset \psi(W \times U)^{n}, \psi$ is bounded on $W \times U^{n}$, hence on sets $W \times S$, where $S$ is a bounded subset of the identity component $G_{1} \subset G$. Further, if $S$ is such and $h \in H$,

$$
\psi\left(h W^{n}, S\right) \subset \psi_{h}\left(\psi _ { W } \left(\psi_{W}\left(\ldots \psi_{W}(S) \ldots\right) ;\right.\right.
$$

the set inside $\psi_{h}$, a subset of $G_{1}$, is bounded by induction. Hence $\psi\left(h W^{n}, S\right)$ is bounded by part (a). It follows that $\psi$ is bounded on sets $K \times S$ with $K \subset H, S \subset G_{1}$ bounded. We still have to show $\psi$ is bounded on sets $K \times g S, K, S$ as above, $g \in G$. Fix $g$. There are sets $K \subset H$ such that $\psi_{K}(g)$ is bounded: any finite set and appropriate neighborhoods of $1_{H}$ will do. If $K$ and $L$ are such sets, $L$ a connected neighborhood of $1_{H}$, then $K L$ is also such a set. Indeed,

$$
\psi_{K L}(g)=\psi_{K}\left(\psi_{L}(g)\right) \subset \psi_{K}(g) \psi_{K}\left(g^{-1} \psi_{L}(g)\right)
$$

is bounded as $g^{-1} \psi_{L}(g) \subset G_{1}$ is bounded. Thus $\psi_{K}(g)$ is bounded for any bounded $K \subset H$. If $S \subset G_{1}$ is also bounded then $\psi_{K}(g S) \subset \psi_{K}(g) \psi_{K}(S)$ is bounded, whence it follows that $\psi$ is indeed bounded on all bounded subsets of $H \times G$. 
To prove uniform continuity, we first claim that, given a neighborhood $U$ of $1_{G}$

(i) for any bounded $K \subset H$ there is a neighborhood $V$ of $1_{G}$ such that $\psi_{h}(g) \in U$, for $(h, g) \in K \times V$

(ii) for any bounded $S \subset G$ there is a neighborhood $W$ of $1_{H}$ such that $g^{-1} \psi_{h}(g) \in U$, for $(h, g) \in W \times S$.

To verify (i) note that it holds for finite $K$, and by Lemma 2.4 also for some neighborhood $K$ of $1_{H}$, independent of $U$. If it holds for $K$ and $L$, it will also hold for $K L$ as $\psi_{k l}(g)=\psi_{k}\left(\psi_{l}(g)\right)$. It follows that (i) indeed holds, and (ii) is proved similarly, using Corollary 2.6, too.

That $\psi$ is uniformly continuous on bounded sets now follows from the identity

$$
\psi_{h}(a)^{-1} \psi_{k}(b)=\psi_{h}\left(a^{-1} b\right) \psi_{h}\left(b^{-1} \psi_{h^{-1} k}(b)\right),
$$

taking (i) and (ii) into account.

If $A$ is a subset of a third Banach-Lie group $\Gamma$ and $S \subset G$, let $C_{u}(A ; S)$ denote the family of bounded, uniformly continuous maps $A \rightarrow S$. Theorems $2.7,2.8 \mathrm{imply}$

Corollary 2.9. (a) $C_{u}(A ; G)$ is closed under pointwise multiplication and inversion.

(b) If $\xi \in C_{u}(A ; \mathfrak{g})$ then $\exp \circ \xi \in C_{u}(A ; G)$. With $U_{0} \subset G, \mathfrak{u}_{0} \subset \mathfrak{g}$ as in Theorem 2.7(c) if $f \in C_{u}\left(A ; U_{0}\right)$ then $\log \circ f \in C_{u}\left(A ; \mathfrak{u}_{0}\right)$

(c) If $\varphi: H \rightarrow G$ is a homomorphism and $f \in C_{u}(A ; H)$ then $\varphi \circ f \in C_{u}(A ; G)$.

(d) If $\psi: H \times G \rightarrow G$ is a smooth action by automorphisms of $G$ and $h \in$ $C_{u}(A ; H), g \in C_{u}(A ; G)$, then $\psi(h, g) \in C_{u}(A ; G)$.

When all groups involved are complex Lie groups, and $A, S$ are open, write $\mathcal{O}_{u}(A ; S)$ for the family of bounded, uniformly continuous holomorphic maps. In this situation we have

Corollary 2.10. The previous corollary is true with $C_{u}$ replaced by $\mathcal{O}_{u}$; (d) under the assumption that $\psi$ is a holomorphic action.

Accordingly, $\mathcal{O}_{u}(A ; G)$ is a group under pointwise multiplication. It can be endowed with the structure of a complex Banach-Lie group as follows. Fixing a norm on $\mathfrak{g}$, the sup norm turns $\mathcal{O}_{u}(A ; \mathfrak{g})$ into a Banach space. Composition with exp and $\log$ defines maps Exp: $\mathcal{O}_{u}(A ; \mathfrak{g}) \rightarrow \mathcal{O}_{u}(A ; G)$ and $\log : \mathcal{O}_{u}\left(A ; U_{0}\right) \rightarrow \mathcal{O}_{u}\left(A ; \mathfrak{u}_{0}\right)$, with $\mathfrak{u}_{0}, U_{0}$ as in Theorem 2.7(c). Choose a neighborhood $\mathfrak{u}_{1}$ of $0 \in \mathfrak{g}$ so that $\left(\exp \mathfrak{u}_{1}\right)^{3} \subset U_{0}$. Then the zero function $0 \in \mathcal{O}_{u}(A ; \mathfrak{g})$ is an interior point of $\mathcal{O}_{u}\left(A ; \mathfrak{u}_{1}\right)$; fix a symmetric open neighborhood $\mathfrak{v} \subset \mathcal{O}_{u}\left(A ; \mathfrak{u}_{1}\right)$ of 0 . With each $f \in \mathcal{O}_{u}(A ; G)$ we associate a chart

$$
\Phi_{f}: \mathfrak{v} \ni \xi \mapsto f \operatorname{Exp} \xi \in \mathcal{O}_{u}(A ; G),
$$

and push forward neighborhoods of $0 \in \mathfrak{v}$ by $\Phi_{f}$ to define a neighborhood basis of $f$. One checks that in this way a Hausdorff topology is obtained on $\mathcal{O}_{u}(A ; G)$. To verify the charts are holomorphically related, take two overlapping charts, say $f \in \Phi_{g}(\mathfrak{v}) \cap \Phi_{h}(\mathfrak{v})$. Thus $f=g \operatorname{Exp} \eta=h \operatorname{Exp} \zeta$, with $\eta, \zeta \in \mathfrak{v}$; the corresponding transition map is given by

$$
\left(\Phi_{g}^{-1} \circ \Phi_{h}\right)(\xi)=\log \left(g^{-1} h \operatorname{Exp} \xi\right)=\log \{\operatorname{Exp} \eta \operatorname{Exp}(-\zeta) \operatorname{Exp} \xi\}
$$

whenever the left hand side is defined, i.e., for $\xi$ in an open set $\mathfrak{w} \subset \mathfrak{v}$. Theorem 2.7 implies $\Phi_{g}^{-1} \circ \Phi_{h}$ is continuous. That it is also holomorphic follows from Morera's 
theorem. Indeed, take a simply connected domain $D \subset \mathbb{C}$, a rectifiable Jordan curve $C \subset D$, and a holomorphic map $D \ni \lambda \mapsto \xi_{\lambda} \in \mathfrak{w}$. The integral of $\left(\Phi_{g}^{-1} \circ \Phi_{h}\right)\left(\xi_{\lambda}\right)$ along $C$ vanishes, for if $a \in A$

$$
\left(\int_{C}\left(\Phi_{g}^{-1} \circ \Phi_{h}\right)\left(\xi_{\lambda}\right) d \lambda\right)(a)=\int_{C} \log \left\{g^{-1}(a) h(a) \exp \xi_{\lambda}(a)\right\} d \lambda=0
$$

(note that for fixed $a$ the second integrand is a holomorphic function of $\lambda$, since the operations on $G, \mathfrak{g}$ are holomorphic). We conclude $\Phi_{g}^{-1} \circ \Phi_{h}$ are holomorphic, and so a complex Banach manifold structure is defined on $\mathcal{O}_{u}(A ; G)$.

The group operations are also holomorphic. For example, to show multiplication is holomorphic near $(g, h) \in \mathcal{O}_{u}(A ; G) \times \mathcal{O}_{u}(A ; G)$ we use the charts $\Phi_{g}, \Phi_{h}, \Phi_{g h}$. In these charts multiplication is given by

$$
(\xi, \eta) \mapsto \log \left\{(g h)^{-1} g(\operatorname{Exp} \xi) h \operatorname{Exp} \eta\right\}=\log \left\{\left(\operatorname{Ad}_{h} \operatorname{Exp} \xi\right) \operatorname{Exp} \eta\right\},
$$

(where now $\operatorname{Ad}_{h}$ stands for the self map of $\mathcal{O}_{u}(A ; G)$ gotten by applying $\operatorname{Ad}_{h(a)}: G \rightarrow$ $G$ pointwise). Since Ad is a holomorphic action of $G$ on itself, Theorems 2.7, 2.8, combined with Morera's theorem, prove as above that (2.3) is holomorphic for $\xi, \eta$ sufficiently small.

In the next section we shall use this construction for subsets $A$ of a Banach space $X$, viewed as an additive group.

3. Uniform bundles. Consider a Banach space $(X,\|\|)$, an open $\Omega \subset X$, and a holomorphic Banach bundle $E \rightarrow \Omega$. For simplicity we assume that all fibers are isomorphic to the same Banach space $Z$, which is certainly true if $\Omega$ is connected. We shall write $\operatorname{Hom}(E, Z)$ for the bundle of homomorphisms between $E$ and the trivial bundle with fiber $Z$, so that the fibers of $\operatorname{Hom}(E, Z)$ are the $\operatorname{spaces} \operatorname{Hom}\left(E_{x}, Z\right)$ of continuous linear maps, $x \in \Omega$. By a uniformization of $E$ we mean a choice of an open covering $\mathfrak{U}$ of $\Omega$ and holomorphic trivializations $e_{U} \in \mathcal{O}(U, \operatorname{Hom}(E, Z)), U \in \mathfrak{U}$, such that $e_{U} e_{V}^{-1} \in \mathcal{O}(U \cap V ; \mathrm{GL}(Z))$ are bounded and uniformly continuous in the sense explained in Section 2. A bundle endowed with such a uniformization will be called uniform or, to be more specific, $\mathfrak{U}$-uniform.

If $X^{\prime}$ is another Banach space, $\Omega \subset X^{\prime}$ open, $\pi: \Omega^{\prime} \rightarrow \Omega$ holomorphic and uniformly continuous, then the induced bundle $\pi^{*} E \rightarrow \Omega^{\prime}$ has a natural uniformization

$$
e_{U}^{\prime}=e_{U} \circ \pi \in \mathcal{O}\left(\pi^{-1} U, \operatorname{Hom}\left(\pi^{*} E, Z\right)\right), \quad U \in \mathfrak{U} .
$$

In particular, the restriction of $E$ to an open subset $\Omega^{\prime} \subset \Omega$, or to $\Omega \cap Y, Y \subset X$ a closed subspace, are both uniform relative the coverings $\mathfrak{U} \mid \Omega^{\prime}$ resp. $\mathfrak{U} \mid Y$. Other natural operations on vector bundles also respect uniform structures: for example, if $E, F \rightarrow \Omega$ are $\mathfrak{U}$-uniform then so will be $E \oplus F$ and $\operatorname{Hom}(E, F)$.

Now fix a complex Banach-Lie group $G$. Uniformization of holomorphic principal $G$-bundles $E \rightarrow \Omega$ is defined analogously.* Writing $\operatorname{Hom}(E, G)$ for the bundle of homomorphisms between $E$ and the trivial $G$-bundle, we are to choose $e_{U} \in$ $\mathcal{O}(U, \operatorname{Hom}(E, G))$ so that the transition functions $e_{U} e_{V}^{-1} \in \mathcal{O}\left(U \cap V, \operatorname{End}_{G} G\right)$ are bounded and uniformly continuous. Here $\operatorname{End}_{G} G$ stands for those maps $G \rightarrow G$ that commute with left translations. These maps are right translations by elements of $G$; thus $\operatorname{End}_{G} G$ is canonically isomorphic with $G$. Again, natural operations on

\footnotetext{
* Our convention is that $G$ acts on $E$ from left.
} 
$G$-bundles respect uniform structures. Here are a few details for $\operatorname{Hom}(E, F)$, if $F$ is a $G$-bundle uniformized by $f_{U}, U \in \mathfrak{U}$. First, $\operatorname{Hom}(E, F)$ is a principal $G$-bundle, the $G$ action given by post-composing $\varphi \in \operatorname{Hom}\left(E_{x}, F_{x}\right)$ with the action of $G$ on $F$. One then checks that by associating with $\varphi$ the endomorphism $h_{U}(\varphi) \in \operatorname{End}_{G} G$

$$
h_{U}(\varphi)(g)=f_{U}(x) \varphi e_{U}^{-1}(x)(g), \quad g \in G, U \ni x,
$$

a system $h_{U}$ of uniformizing sections is obtained.

There is an obvious connection between uniformized Banach and principal bundles. If $E$ is a uniformized Banach bundle with fibers isomorphic to $Z$ then the bundle $\operatorname{Iso}(E, Z) \subset \operatorname{Hom}(E, Z)$ of isomorphisms is a principal $\operatorname{GL}(Z)$-bundle with a natural uniformization. Conversely, if $E$ is a uniformized principal $G$-bundle and $\pi: G \rightarrow \mathrm{GL}(Z)$ a holomorphic representation, then the associated Banach bundle $E^{\pi}$ has a natural uniformization.

A homomorphism $\varphi: E \rightarrow F$ between holomorphic principal $G$-bundles uniformized by $e_{U}$ resp. $f_{U}, U \in \mathfrak{U}$, is called uniform if $f_{U} \varphi e_{U}^{-1} \in \mathcal{O}_{u}\left(U ; \operatorname{End}_{G} G\right)$ for all $U$. In view of Corollaries 2.9, 2.10, the inverse of such a homomorphism is also uniform, hence we also call $\varphi$ a uniform isomorphism. If such $\varphi: E \rightarrow F$ exists, we say $E$ and $F$ are uniformly isomorphic. An isomorphism $\psi: E \rightarrow F$ between $\mathfrak{U}$-uniform holomorphic Banach bundles is called uniform if the associated map

$$
\operatorname{Iso}(F, Z) \rightarrow \operatorname{Iso}(E, Z)
$$

of $\mathfrak{U}$-uniform principal bundles is a uniform isomorphism.

For sections $f$ of a principal or Banach bundle $E \rightarrow \Omega$ uniformized by trivializations $e_{U}$ one can introduce the notions of boundedness and uniform continuity: the condition will be that $e_{U} f: U \rightarrow G$ (resp. $Z$ ) should be bounded resp. uniformly continuous for all $U$. We shall denote the space of bounded, uniformly continuous holomorphic sections by $\mathcal{O}_{u}(\Omega, E)$. For a uniformized Banach bundle $E \rightarrow \Omega$ and a subset $\Omega_{0} \subset \Omega$ one can also introduce the topology of $\mathfrak{U}$-local uniform convergence on $\mathcal{O}\left(\Omega_{0}, E\right)$, the space of germs of holomorphic sections at $\Omega_{0}$. Basic neighborhoods of $f \in \mathcal{O}\left(\Omega_{0}, E\right)$ will be labeled by positive functions $\varepsilon$ defined on some finite $\mathfrak{V} \subset \mathfrak{U}$ : the neighborhood corresponding to $\varepsilon$ is

$$
\left\{g \in \mathcal{O}\left(\Omega_{0}, E\right): \sup _{V \cap \Omega_{0}}\left\|e_{V}(g-f)\right\|_{Z}<\varepsilon(V) \text { for all } V \in \mathfrak{V}\right\} .
$$

We emphasize that both the space $\mathcal{O}_{u}(\Omega, E)$ and the topology on $\mathcal{O}\left(\Omega_{0}, E\right)$ depend on the choice of the uniformization.-It follows from Theorems 2.7, 2.8 that a uniform isomorphism $\varphi: E \rightarrow F$ induces a topological isomorphism $\mathcal{O}\left(\Omega_{0}, E\right) \rightarrow \mathcal{O}\left(\Omega_{0}, F\right)$.

Further down we shall use the following terminology. Let $\Omega_{0} \subset \Omega_{1}, \Omega_{2} \subset \Omega$.

Definition 3.1. We say that $\mathcal{O}\left(\Omega_{2}, E\right)$ is dense in $\mathcal{O}\left(\Omega_{1}, E\right)$ over $\Omega_{0}$ if $\mathcal{O}\left(\Omega_{2}, E\right) \mid \Omega_{0}$ is dense in $\mathcal{O}\left(\Omega_{1}, E\right) \mid \Omega_{0}$, in the topology of $\mathcal{O}\left(\Omega_{0}, E\right)$.

Loosely speaking, this means that a certain Runge type approximation is possible.

In this section we shall study uniform bundles over certain ball bundles, and prove a (weak) instance of the Grauert-Oka principle: two uniform Banach resp. principal bundles, that are easily seen to be topologically isomorphic, are in fact holomorphically isomorphic. From this we shall derive an approximation and a cohomology vanishing result, Corollary 3.5. 
The ball bundles in question are gotten as follows. Let $\pi: X \rightarrow X$ be a projection on a finite dimensional subspace and $\rho=\operatorname{id}_{X}-\pi$ the complementary projection. Given $A \subset \pi X$ and $r: A \rightarrow(0, \infty)$ continuous, the sets

$$
\begin{gathered}
A(r)=\{x \in X: \pi x \in A,\|\rho x\|<r(\pi x)\}, \\
A[r]=\{x \in X: \pi x \in A,\|\rho x\| \leq r(\pi x)\}
\end{gathered}
$$

are called sets of type (B) (with respect to $\pi$ ). By [Lm2, Proposition 4.1] $A(r)$ is open and pseudoconvex precisely when $A$ is open and pseudoconvex, and $-\log r$ is plurisubharmonic. To any open covering $\mathfrak{V}$ of $A$ there corresponds a covering

$$
\mathfrak{V}(r)=\{V(r): V \in \mathfrak{V}\}
$$

of $A(r)$. With this notation we have

THEOREM 3.2. Suppose $A(r)$ is open and pseudoconvex. Let $\mathfrak{V}$ be an open covering of $A$ and $E \rightarrow A(r)$ a $\mathfrak{V}(r)$-uniform holomorphic Banach or principal $G$-bundle. If $A_{1} \subset \subset A$ is open and $r_{1}: A_{1} \rightarrow(0, \infty)$ continuous, $r_{1}<r \mid A_{1}$, then $\pi^{*} E \mid A_{1}\left(r_{1}\right)$ and $E \mid A_{1}\left(r_{1}\right)$ are uniformly isomorphic.

The theorem will be deduced from an analogous but finite dimensional result concerning bundles on which $S^{1}$ acts. Let $\Delta \subset \mathbb{C}$ denote the open unit disc, $\bar{\Delta}$ its closure; $S^{1}=\bar{\Delta} \backslash \Delta$. The multiplicative semigroup $\bar{\Delta}$ acts on $A(r)$ by

$$
g_{\omega} x=\pi x+\omega \rho x, \quad \omega \in \bar{\Delta}, x \in A(r) .
$$

If $A(r)$ is open, the restriction of the action to $\Delta$ is holomorphic.

Proposition 3.3. Suppose $\operatorname{dim} X<\infty$ and $A(r)$ is open and pseudoconvex. Let $F \rightarrow A(r), H \rightarrow A(r)$ be locally trivial holomorphic principal $G$-bundles, and suppose the $S^{1}$ action $\left\{g_{\omega}: \omega \in S^{1}\right\}$, cf. (3.2), lifts to continuous actions both on $F$ and $H$, by biholomorphic $G$-maps. If there is an $S^{1}$ equivariant isomorphism $\psi_{0}: F|A \rightarrow H| A$ then there is an equivariant isomorphism $\psi: F \rightarrow H$.

Proof. First construct an $S^{1}$ invariant holomorphic connection $\nabla^{F}$ on $F$. Since $F$ is locally trivial, $\Omega=A(r)$ can be covered by open subsets $U$ so that each $F \mid U$ admits a holomorphic connection $\nabla_{U}$. The difference $\nabla_{U}-\nabla_{V}$ of two such local connections can be represented by a holomorphic one form on $U \cap V$, with values in a Banach bundle $\mathfrak{f}$ (the adjoint Lie algebra bundle), or by a section $\alpha_{U V}$ of $\mathfrak{f} \otimes T^{*} \Omega$ over $U \cap V$. Since $H^{1}(\Omega, E)=0$ for any locally trivial holomorphic Banach bundle $E \rightarrow \Omega$ by [Li, Theorem 2.3], there are $\alpha_{U} \in \mathcal{O}\left(U, \mathfrak{f} \otimes T^{*} \Omega\right)$ such that $\alpha_{U V}=\alpha_{U}-\alpha_{V}$. Then $\nabla=\nabla_{U}-\alpha_{U}$ over $U$ consistently defines a holomorphic connection on $F$. To achieve $S^{1}$ equivariance, let $d \omega$ denote the Haar probability measure on $S^{1}$. The space $\mathcal{C}$ of holomorphic connections on $F$ (with the compact open topology) being an affine space of a Fréchet space, continuous $\mathcal{C}$-valued functions can be integrated against Radon probability measures. Hence

$$
\nabla^{F}=\int_{S^{1}}\left(\omega^{*} \nabla\right) d \omega
$$

defines a holomorphic connection on $F$ that is easily seen to be $S^{1}$ invariant.

Similarly, there is an invariant holomorphic connection $\nabla^{H}$ on $H$. Now construct $\psi$ as follows. If $x \in A(r)$ and $v \in F_{x}$, in $\left(F, \nabla^{F}\right)$ parallel transport $v$ along the 
segment $[x, \pi x]$, to obtain $v_{0} \in F_{\pi x}$. Next in $\left(H, \nabla^{H}\right)$ parallel transport $\psi_{0}\left(v_{0}\right)$ along the segment $[\pi x, x]$; the vector thus gotten will be $\psi(v) \in H_{x}$. Since all ingredients of this construction are holomorphic and equivariant, $\psi$ will indeed be an equivariant isomorphism.

Now consider a principal $G$-bundle $E \rightarrow A(r)$ as in Theorem 3.2. We shall assume $r$ is bounded, without loss of generality. Introduce the finite dimensional Banach space $X^{\prime}=\pi X \oplus \mathbb{C}$ and the Hartogs set

$$
A(r)^{\prime}=\{(\xi, \eta) \in A \times \mathbb{C}:|\eta|<r(\xi)\} \subset X^{\prime},
$$

cf. (3.1), and define $V(r)^{\prime} \subset A(r)^{\prime}$ similarly for $V \in \mathfrak{V}$. We shall apply a Radon type transformation to $E$ to obtain a $\mathfrak{V}(r)^{\prime}=\left\{V(r)^{\prime}: V \in \mathfrak{V}\right\}$-uniform bundle $P E=F \rightarrow$ $A(r)^{\prime}$ as follows. Let $B$ denote the unit ball in $\operatorname{Ker} \pi \subset X$, and consider the double fibration

$$
\begin{aligned}
& A(r)^{\prime} \times B \\
& a \swarrow \searrow \alpha \\
& A(r) \quad A(r)^{\prime},
\end{aligned}
$$

where $a(\zeta, y)=\xi+\eta y$ for $\zeta=(\xi, \eta) \in A(r)^{\prime}, y \in B$, and $\alpha$ is projection on the first factor. The bundle $F$ will be defined as the direct image of $a^{*} E$ under $\alpha$.

Concretely, set $a_{\zeta}=a(\zeta, \cdot)$,

$$
F_{\zeta}=\mathcal{O}_{u}\left(B, a_{\zeta}^{*} E\right), \quad \text { and } \quad F=\amalg_{\zeta \in A(r)} F_{\zeta} .
$$

If $e_{V} \in \mathcal{O}(V(r), \operatorname{Hom}(E, G))$ are the uniformizing trivializations of $E$, the maps

$$
f_{V}(\zeta): \mathcal{O}_{u}\left(B, a_{\zeta}^{*} E\right) \ni s \mapsto\left(a_{\zeta}^{*} e_{V}\right) s \in \mathcal{O}_{u}(B ; G), \quad \zeta \in V(r)^{\prime},
$$

endow $F=P E \rightarrow A(r)^{\prime}$ with the structure of a locally trivial holomorphic principal bundle of the group $P G=\mathcal{O}_{u}(B ; G)$. Since for $\zeta \in U(r)^{\prime} \cap V(r)^{\prime}$ the transition functions $f_{U}(\zeta) f_{V}(\zeta)^{-1} \in \operatorname{End}_{P G} P G$ are defined by applying $a_{\zeta}^{*}\left(e_{U} e_{V}^{-1}\right) \in$ $\mathcal{O}_{u}\left(B ; \operatorname{End}_{G} G\right)$, the trivializations uniformize $F$.

The semigroup $\bar{\Delta}$ acts on $A(r)^{\prime}$ and $B$ by $\gamma_{\omega}(\xi, \eta)=(\xi, \omega \eta)$ and $\beta_{\omega}(y)=\omega y, \omega \in$ $\bar{\Delta}$. Restricting for the moment to $S^{1} \subset \bar{\Delta}$, all spaces in (3.3) acquire an $S^{1}$ action. For $A(r)^{\prime}$ this is $\gamma \mid S^{1}$, for $A(r)$ the trivial action, and for $A(r)^{\prime} \times B$ the action $\left(\gamma \mid S^{1}\right) \times$ $\left(\beta \mid S^{1}\right)^{-1}$; both maps $a$ and $\alpha$ are equivariant. The trivial action on $A(r)$ lifts to the trivial action on $E$, which gives rise to an $S^{1}$ action on $a^{*} E$, and also on $F$ as follows. Since

$$
a_{\gamma_{\omega} \zeta}=a_{\zeta} \beta_{\omega}, \quad \text { and so } \quad a_{\gamma_{\omega} \zeta}^{*}=\beta_{\omega}^{*} a_{\zeta}^{*}
$$

pull back by $\beta_{\omega}$ induces an isomorphism

$$
\beta_{\omega}^{*}: F_{\zeta}=\mathcal{O}_{u}\left(B, a_{\zeta}^{*} E\right) \rightarrow F_{\gamma_{\omega} \zeta}=\mathcal{O}_{u}\left(B, a_{\gamma_{\omega} \zeta}^{*} E\right)
$$

Thus the $S^{1}$ action $\beta_{\omega}^{*}$ on $F$ covers the action $\gamma_{\omega}$ on $A(r)^{\prime}$.

There is a monomorphism

$$
P: \mathcal{O}_{u}(A(r), E) \rightarrow \mathcal{O}_{u}\left(A(r)^{\prime}, P E\right)
$$


given by $(P e)(\zeta)=a_{\zeta}^{*} e$. Using (3.4) one checks that $P e$ is $S^{1}$ equivariant for all $e \in \mathcal{O}_{u}(A(r), E)$ (which is not surprising, since $e$ itself was equivariant, the actions on $A(r), E$ being trivial). Conversely, we have

Proposition 3.4. Any $S^{1}$ equivariant $f \in \mathcal{O}_{u}\left(A(r)^{\prime}, P E\right)$ is of form $f=P e, e \in$ $\mathcal{O}_{u}(A(r), E)$.

Proof. Equivariance means

$$
\beta_{\omega}^{*} f(\zeta)=f\left(\gamma_{\omega} \zeta\right), \quad \omega \in S^{1}
$$

As both sides depend continuously on $\omega \in \bar{\Delta}$, indeed holomorphically when $\omega \in \Delta$, unique continuation implies (3.5) holds for all $\omega \in \bar{\Delta}$. In other words, if $\zeta^{\prime}=\gamma_{\omega} \zeta, y=$ $\beta_{\omega} y^{\prime}$ with $\omega \in \bar{\Delta}$ then $f(\zeta)(y)=f\left(\zeta^{\prime}\right)\left(y^{\prime}\right)$. This implies that if for $A(r) \ni x=a(\zeta, y)$ we define $e(x)=f(\zeta)(y) \in E_{x}$ then $e(x)$ is independent of the choice of $(\zeta, y) \in$ $A(r)^{\prime} \times B$. Thus we obtain a section $e \in \mathcal{O}_{u}(A(r), E)$; clearly $P e=f$ as needed.

Proof of Theorem 3.2. We shall only deal with principal bundles $E$, since the part about Banach bundles will follow upon passing to the associated principal bundle. Applying the transformation $P$ to various bundles we claim that

$$
P \operatorname{Hom}\left(\pi^{*} E, E\right) \approx \operatorname{Hom}\left(P \pi^{*} E, P E\right)
$$

as $P G$-bundles with $S^{1}$ actions. Indeed, if $\zeta \in A(r)^{\prime}$, any

$$
\varphi \in \mathcal{O}_{u}\left(B, a_{\zeta}^{*} \operatorname{Hom}\left(\pi^{*} E, E\right)\right)=\left.P \operatorname{Hom}\left(\pi^{*} E, E\right)\right|_{\zeta}
$$

induces, by pointwise post-composition, a

$$
\Phi \in \operatorname{Hom}\left(\mathcal{O}_{u}\left(B, a_{\zeta}^{*} \pi^{*} E\right), \mathcal{O}_{u}\left(B, a_{\zeta}^{*} E\right)\right)=\left.\operatorname{Hom}\left(P \pi^{*} E, P E\right)\right|_{\zeta} ;
$$

the map $\varphi \mapsto \Phi$ defines an $S^{1} \times P G$ equivariant map from the left hand side of (3.6) to the right hand side, that covers $\gamma \mid S^{1} \times \operatorname{id}_{A(r)^{\prime}}$. It follows that this map realizes the isomorphism in (3.6).

Now $\pi^{*} E|A=E| A$. Hence $P \pi^{*} E|A \times\{0\}=P E| A \times\{0\}$, and the identity is an $S^{1}$ equivariant homomorphism between these bundles. By Proposition 3.3 there is therefore an $S^{1}$ equivariant homomorphism

$$
f \in \mathcal{O}\left(A(r)^{\prime}, \operatorname{Hom}\left(P \pi^{*} E, P E\right)\right) \approx \mathcal{O}\left(A(r)^{\prime}, P \operatorname{Hom}\left(\pi^{*} E, E\right)\right) .
$$

Although $f$ may not be bounded and uniformly continuous, $f_{1}=f \mid A_{1}\left(r_{1}\right)^{\prime}$ certainly is. Hence by Proposition $3.4 f_{1}=P e_{1}$ with some uniform homomorphism $e_{1}: \pi^{*} E\left|A_{1}\left(r_{1}\right) \rightarrow E\right| A_{1}\left(r_{1}\right)$, and the theorem follows.

Corollary 3.5. Suppose $X$ has a Schauder basis and Hypothesis 1.2 holds with some $\mu$. Let $A(r) \subset X$ be open and pseudoconvex, $E \rightarrow A(r)$ a $\mathfrak{V}(r)$-uniformized holomorphic Banach bundle. If $A_{1} \subset \subset A$ is open, $r_{1}: A_{1} \rightarrow(0, \infty)$ continuous, $r_{1}<$ $r \mid A_{1}$, and $A_{1}\left(r_{1}\right)$ pseudoconvex, then

(a) $H^{q}\left(A_{1}\left(r_{1}\right), E\right)=0$ for $q \geq 1$.

(b) Suppose furthermore that $A_{0} \subset A_{1} \subset \subset A_{2} \subset \subset A$, with $A_{0}$ compact and plurisubharmonically convex in $A, A_{2}$ open; $r_{i}: A_{i} \rightarrow(0, \infty)$ are continuous with $r_{0}<$ $\mu r_{1}\left|A_{0}, r_{1}<r\right| A_{1}, r_{2}<r \mid A_{2}$. Then $\mathcal{O}\left(A_{2}\left(r_{2}\right), E\right)$ is dense in $\mathcal{O}\left(A_{1}\left(r_{1}\right), E\right)$ over $A_{0}\left[r_{0}\right]$. 
Proof. We shall follow [P1, sections 4-6]. At the price of shrinking $A(r)$ we can assume $\pi^{*} E \mid A(r)$ and $E$ are uniformly isomorphic, by Theorem 3.2; and also that there is a locally trivial holomorphic Banach bundle $F \rightarrow A$ with $F \oplus E \mid A$ isomorphic to a trivial bundle $T \rightarrow A$, by [P1, Proposition 4.1]. Then $\left(\pi^{*} F \oplus E\right) \mid A_{1}\left(r_{1}\right)$ is isomorphic to the trivial bundle $\pi^{*} T \mid A_{1}\left(r_{1}\right)$, so that $H^{q}\left(A_{1}\left(r_{1}\right), E\right)$ embeds in $H^{q}\left(A_{1}\left(r_{1}\right), \pi^{*} T\right)$. Since this latter vanishes by [Lm2, Theorem 0.3], (a) follows.

To prove (b) note that $F$ will be uniform for any covering $\mathfrak{W}$ of $A$ by open $W \subset \subset A$. If $\mathfrak{W}$ is a refinement of $\mathfrak{V}$ then the $\mathfrak{V}(r)$-uniformization of $E$ will induce a $\mathfrak{W}(r)$-uniformization; moreover, the topologies on $\mathcal{O}\left(A_{0}\left[r_{0}\right], E\right)$ induced by the two uniformizations will coincide. The trivial bundle $T$ also has a natural $\mathfrak{W}-$ uniformization. After some more shrinking the $\mathfrak{W}$-uniform bundles $F \oplus E \mid A$ and $T$ will be uniformly isomorphic, and so will be the induced $\mathfrak{W}(r)$-uniform bundles $\pi^{*} F \mid A(r) \oplus E$ and $\pi^{*} T \mid A(r)=T^{\prime}$. By Theorem 6.1 in [Lm2] $\mathcal{O}\left(A_{2}\left(r_{2}\right), T^{\prime}\right)$ is dense in $\mathcal{O}\left(A_{1}\left(r_{1}\right), T^{\prime}\right)$ over $A_{0}\left[r_{0}\right]$, which implies (b).

4. Exhaustion. All through this section we shall work with a Banach space $(X,\|\|)$ that has a Schauder basis $e_{1}, e_{2}, \ldots$, and satisfies Hypothesis 1.2 with some $\mu ; \Omega \subset X$ will be open and pseudoconvex, and $E \rightarrow \Omega$ a $\mathfrak{U}$-uniform holomorphic Banach bundle with fibers isomorphic to the Banach space $Z$. We shall exhaust $\Omega$ by open sets $\Omega_{N}$ of type (B) in a way adapted to $\mathfrak{U}$, discuss properties of $\Omega_{N}$, and prove two approximation results, Theorem 4.5 and Proposition 4.6.

Introduce projections $\pi_{N}: X \rightarrow X$

$$
\pi_{N} \sum_{1}^{\infty} \lambda_{j} e_{j}=\sum_{1}^{N} \lambda_{j} e_{j}, \quad \lambda_{j} \in \mathbb{C}, \quad \pi_{0}=0, \quad \pi_{\infty}=\operatorname{id}_{X}
$$

and $\rho_{N}=\operatorname{id}_{X}-\pi_{N}$. We shall assume for all $x \in X$

$$
\left\|\pi_{N} x-\pi_{M} x\right\| \leq\left\|\pi_{n} x-\pi_{m} x\right\|, \quad 0 \leq n \leq N \leq M \leq m \leq \infty .
$$

As pointed out in [Lm2, Section 7], this can be arranged upon equivalently renorming $X$.

Definition 4.1. A continuous function $\alpha: \Omega \rightarrow(0,1)$ is adapted (to $\mathfrak{U}$ ) if

(i) $\alpha(x)<\operatorname{dist}_{X \backslash \Omega}(x)$ for $x \in \Omega$;

(ii) the covering $\{B(x, \alpha(x)): x \in \Omega\}$ is finer than $\mathfrak{U}$;

(iii) $-\log \alpha$ is plurisubharmonic; and

(iv) in case $\Omega$ is unbounded, $\lim _{\|x\| \rightarrow \infty} \alpha(x)=0$.

Denote the family of adapted functions $\mathcal{A}=\mathcal{A}_{\mathfrak{U}}$. It is clear that if $\alpha, \beta \in \mathcal{A}$ and $c \in(0,1)$, then $c \alpha, \min \{\alpha, \beta\} \in \mathcal{A}$. What is not obvious is whether there are adapted functions at all.

Lemma 4.2. $\mathcal{A} \neq \emptyset$.

Proof. For $x \in \Omega$ let $\beta(x)=\sup \{r \in(0,1): B(x, r) \subset U$ with some $U \in \mathfrak{U}\}$ and

$$
u(x)=\max \left\{-\log \beta(x),-\log \operatorname{dist}_{X \backslash \Omega}(x),\|x\|\right\} .
$$

As $u: \Omega \rightarrow \mathbb{R}$ is locally bounded, by [Lm4, Theorem 1.6] there is a continuous plurisubharmonic $v>u$ on $\Omega$; then $e^{-v} \in \mathcal{A}$. 

define

Fix $c \in(0,1)$. If $\alpha: \Omega \rightarrow(0,1)$ is continuous, $\alpha<\operatorname{dist}_{X \backslash \Omega}$, and $N=0,1, \ldots$,

$$
\begin{aligned}
& D_{N}\langle\alpha\rangle=\left\{\xi \in \Omega \cap \pi_{N} X:(N+1) \alpha(\xi)>c\right\}, \\
& \bar{D}_{N}\langle\alpha\rangle=\left\{\xi \in \Omega \cap \pi_{N} X:(N+1) \alpha(\xi) \geq c\right\}, \\
& \Omega_{N}\langle\alpha\rangle=\left\{x \in \pi_{N}^{-1} D_{N}\langle\alpha\rangle:\left\|\rho_{N} x\right\|<\alpha\left(\pi_{N} x\right)\right\}, \\
& \bar{\Omega}_{N}\langle\alpha\rangle=\left\{x \in \pi_{N}^{-1} \bar{D}_{N}\langle\alpha\rangle:\left\|\rho_{N} x\right\| \leq \alpha\left(\pi_{N} x\right)\right\} .
\end{aligned}
$$

We could work with any concrete value of $c$, say $c=1 / 2$, except that in the proof of Theorem 4.5 it will be convenient to choose a $c$ that matches the geometric situation considered.

Thus $\Omega_{N}\langle\alpha\rangle, \bar{\Omega}_{N}\langle\alpha\rangle \subset \Omega$ are sets of type (B) with base $D_{N}\langle\alpha\rangle, \bar{D}_{N}\langle\alpha\rangle$. They are related to the exhausting sets used in [Lm2, Lm4, P1, P2], but previously $\alpha$ was taken essentially a constant multiple of $\operatorname{dist}_{X \backslash \Omega}$. Such $\alpha$ is adapted to very special coverings only, which is, in a way, the reason why [Lm2, P1, P2] had to settle for weaker vanishing theorems.

Proposition 4.3. If $\alpha \in \mathcal{A}$ then

(a) $D_{N}\langle\alpha\rangle \subset \pi_{N} X$ and $\Omega_{N}\langle\alpha\rangle \subset X$ are open, bounded, and pseudoconvex;

(b) there is a $\gamma \in \mathcal{A}$ such that $B(x, \gamma(x)) \subset \Omega_{M}\langle\alpha\rangle$ whenever $x \in \bar{\Omega}_{N}\langle\gamma\rangle, M \geq N$ (in particular, $\bar{\Omega}_{N}\langle\gamma\rangle \subset \Omega_{M}\langle\alpha\rangle$ );

(c) every $x \in \Omega$ has a neighborhood contained in all but finitely many $\Omega_{M}\langle\alpha\rangle$;

(d) if also $4 \alpha \in \mathcal{A}$ then $Y \cap \bar{\Omega}_{N}\langle\alpha\rangle$ is plurisubharmonically convex in $Y \cap \Omega$ for all finite dimensional subspaces $Y \subset X$.

Proof. (a) Openness and boundedness are obvious, and so is pseudoconvexity, since the inequalities in (4.2) describe sublevel sets of functions plurisubharmonic in $\Omega \cap \pi_{N} X$ resp. $\pi_{N}^{-1} D_{N}\langle\alpha\rangle$.

(b) For every $z \in \Omega$ choose $0<\varepsilon_{z}<\alpha(z)$ so that $2 \alpha(z)>\alpha>\alpha(z) / 2$ hold on $B\left(z, \varepsilon_{z}\right)$, making sure that $\log \varepsilon_{z}$ is a locally bounded function of $z$. Plurisubharmonic domination, [Lm4, Theorem 1.6], implies there is a $\beta \in \mathcal{A}$ such that $\beta(z)<\varepsilon_{z} / 2<$ $\alpha(z) / 2$. Thus

$$
2 \alpha(z)>\alpha>\alpha(z) / 2 \quad \text { on } B(z, 2 \beta(z)) .
$$

Similarly, there is $\gamma \in \mathcal{A}, \gamma<\beta / 4$, such that

$$
2 \beta(z)>\beta>\beta(z) / 2 \quad \text { on } B(z, 2 \gamma(z)) .
$$

Now suppose $N, M, x$ are as in the proposition. Since $x \in B\left(\pi_{N} x, 2 \gamma\left(\pi_{N} x\right)\right)$,

$$
\beta(x)>\beta\left(\pi_{N} x\right) / 2>2 \gamma\left(\pi_{N} x\right) .
$$

Therefore, if $y \in B(x, \gamma(x))$, using also (4.1)

$$
\begin{aligned}
\left\|x-\pi_{M} y\right\| & \leq\left\|x-\pi_{M} x\right\|+\left\|\pi_{M}(x-y)\right\| \\
& \leq\left\|x-\pi_{N} x\right\|+\|x-y\|<\gamma\left(\pi_{N} x\right)+\gamma(x)<\beta(x), \text { whence } \\
\alpha\left(\pi_{M} y\right)> & \alpha(x) / 2>\beta(x)>\gamma\left(\pi_{N} x\right) \geq c /(N+1) \geq c /(M+1),
\end{aligned}
$$

and so $\pi_{M} y \in D_{M}\langle\alpha\rangle$. In view of (4.1), (4.2), (4.3), and (4.4)

$$
\left\|\rho_{M} y\right\| \leq\left\|\rho_{N} y\right\| \leq\left\|\rho_{N} x\right\|+\left\|\rho_{N}(x-y)\right\|<\gamma\left(\pi_{N} x\right)+\gamma(x)<\beta(x)<\alpha\left(\pi_{M} y\right) .
$$


Thus $y \in \Omega_{M}\langle\alpha\rangle$, which proves the claim.

(c) Let $\gamma$ be as in (b). A glance at (4.2) shows $x \in \Omega_{N}\langle\gamma\rangle$ for some $N$. By (b) $B(x, \gamma(x))$ is then contained in all $\Omega_{M}\langle\alpha\rangle, M \geq N$.

(d) The function

$$
u(x)=\frac{\max \left\{\left\|\rho_{N} x\right\|, c /(N+1)\right\}}{\alpha\left(\pi_{N} x\right)}, \quad x \in Y \cap \pi_{N}^{-1} \Omega,
$$

is plurisubharmonic - since its logarithm is-, and $Y \cap \bar{\Omega}_{N}\langle\alpha\rangle=\{x: u(x) \leq 1\}$. Further, set $d(x)=\min \left\{1, \operatorname{dist}_{X \backslash \Omega}(x)\right\}, x \in X$, and

$$
v(x)=2 \frac{\max \left\{\left\|\rho_{N} x\right\|, c /(N+1)\right\}}{d(x)}, \quad x \in Y \cap \Omega .
$$

Since $-\log d$ is plurisubharmonic in $\Omega$, see [N], $v$ is plurisubharmonic. When $x \in$ $\bar{\Omega}_{N}\langle\alpha\rangle$

$$
d\left(\pi_{N} x\right) \leq d(x)+\left\|x-\pi_{N} x\right\| \leq d(x)+\alpha\left(\pi_{N} x\right) \leq d(x)+d\left(\pi_{N} x\right) / 4
$$

in view of (4.2) and Definition 4.1; hence $2 d(x)>d\left(\pi_{N} x\right)>4 \alpha\left(\pi_{N} x\right)$. Therefore $v<u \leq 1$ on $Y \cap \bar{\Omega}_{N}\langle\alpha\rangle$. Fix $\varepsilon>0$ so that the plurisubharmonic function $w(x)=$ $v(x)+\varepsilon\|x\|, x \in Y \cap \Omega$, still satisfies

$$
w<1 \quad \text { on } Y \cap \bar{\Omega}_{N}\langle\alpha\rangle .
$$

We claim that if $w(x) \leq 1$ then $x$ is contained in

$$
G=\left\{x \in Y \cap \Omega \cap \pi_{N}^{-1} \Omega: d(x), d\left(\pi_{N} x\right)>c /(N+1),\|x\|<2 / \varepsilon\right\} .
$$

Indeed, (4.5) implies $d(x) \geq 2 c /(N+1)$ and $\left\|\rho_{N} x\right\|<d(x) / 2$, whence $d\left(\pi_{N} x\right) \geq$ $d(x)-\left\|\pi_{N} x-x\right\|>d(x) / 2 \geq c /(N+1)$; the last inequality in (4.7) is obvious.

The boundary of $G$ relative to $Y$, denoted $\partial G$, being compact, $\min _{\partial G} w>1$. One can therefore choose a convex increasing function $\chi: \mathbb{R} \rightarrow \mathbb{R}$ such that $\chi(\xi)=\xi$ for $\xi \leq 1$ and $\chi\left(\min _{\partial G} w\right)>\max _{\partial G} u$. The function

$$
u_{0}= \begin{cases}\max \{u, \chi \circ w\} & \text { on } G \\ \chi \circ w & \text { on } Y \cap \Omega \backslash G .\end{cases}
$$

is plurisubharmonic, since $\chi \circ w$ is plurisubharmonic on $Y \cap \Omega, u$ is plurisubharmonic on $G$, and $\chi \circ w>u$ in a neighborhood of $\partial G$. If $u_{0}(x) \leq 1$ then $x \in G$, whence $u(x) \leq 1$. Conversely, if $u(x) \leq 1$, i.e. $x \in Y \cap \bar{\Omega}_{N}\langle\alpha\rangle$, then $w(x)<1$ by (4.6) and $u_{0}(x) \leq 1$. The upshot is that $Y \cap \bar{\Omega}_{N}\langle\alpha\rangle=\left\{x \in Y \cap \Omega: u_{0}(x) \leq 1\right\}$ is plurisubharmonically convex.

As in [Lm2, Lm4], we shall need another sequence of exhausting sets $\Omega^{N}\langle\alpha\rangle$, each of type (B) but now with respect to the projection $\pi_{N+1}$; at the same time $\Omega^{N}\langle\alpha\rangle$ and $\Omega_{N}\langle\alpha\rangle$ will be comparable. Let

$$
\begin{aligned}
& D^{N}\langle\alpha\rangle=\pi_{N+1} X \cap \Omega_{N}\langle\alpha\rangle, \Omega^{N}\langle\alpha\rangle=\left\{x \in \pi_{N+1}^{-1} D^{N}\langle\alpha\rangle:\left\|\rho_{N+1} x\right\|<\alpha\left(\pi_{N} x\right)\right\}, \\
& \bar{D}^{N}\langle\alpha\rangle=\pi_{N+1} X \cap \bar{\Omega}_{N}\langle\alpha\rangle, \bar{\Omega}^{N}\langle\alpha\rangle=\left\{x \in \pi_{N+1}^{-1} \bar{D}^{N}\langle\alpha\rangle:\left\|\rho_{N+1} x\right\| \leq \alpha\left(\pi_{N} x\right)\right\} .
\end{aligned}
$$

As above, $D^{N}\langle\alpha\rangle$ and $\Omega^{N}\langle\alpha\rangle$ are pseudoconvex if $\alpha \in \mathcal{A}$. 
Proposition 4.4. If $\alpha \in \mathcal{A}$ then $\bar{\Omega}_{N}\langle\alpha\rangle \subset \bar{\Omega}^{N}\langle\alpha\rangle$. If $2 \alpha \in \mathcal{A}$ then $\Omega^{N}\langle\alpha\rangle \subset$ $\Omega_{N}\langle 2 \alpha\rangle$.

Proof. Suppose $x \in \bar{\Omega}_{N}\langle\alpha\rangle, \alpha \in \mathcal{A}$. To check $\pi_{N+1} x=\xi \in \bar{D}^{N}\langle\alpha\rangle$, note that $\pi_{N} \xi=\pi_{N} x \in \bar{D}_{N}\langle\alpha\rangle$ and $\left\|\rho_{N} \xi\right\|=\left\|\pi_{N+1} \rho_{N} x\right\| \leq\left\|\rho_{N} x\right\| \leq \alpha\left(\pi_{N} x\right)$. Thus $\xi \in$ $\bar{\Omega}_{N}\langle\alpha\rangle$, whence $\xi \in \bar{D}^{N}\langle\alpha\rangle$. Further $\left\|\rho_{N+1} x\right\| \leq\left\|\rho_{N} x\right\| \leq \alpha\left(\pi_{N} x\right)$; therefore $x \in$ $\bar{\Omega}^{N}\langle\alpha\rangle$.

Next suppose $x \in \Omega^{N}\langle\alpha\rangle, 2 \alpha \in \mathcal{A}$. Then by (4.8)

$$
\pi_{N} x=\pi_{N} \pi_{N+1} x \in \pi_{N} D^{N}\langle\alpha\rangle \subset \pi_{N} X \cap \pi_{N} \Omega_{N}\langle\alpha\rangle=D_{N}\langle\alpha\rangle .
$$

Since $\rho_{N} x=\rho_{N+1} x+\rho_{N} \pi_{N+1} x$ and $\pi_{N+1} x \in D^{N}\langle\alpha\rangle \subset \Omega_{N}\langle\alpha\rangle$,

$$
\left\|\rho_{N} x\right\|<\left\|\rho_{N+1} x\right\|+\alpha\left(\pi_{N} \pi_{N+1} x\right)<2 \alpha\left(\pi_{N} x\right),
$$

so that $x \in \Omega_{N}\langle 2 \alpha\rangle$.

Now we turn to approximation:

TheOREM 4.5. There is a $\gamma \in \mathcal{A}$ with the following property. Given $N=0,1, \ldots$, suppose $A_{1} \subset \subset \Omega \cap \pi_{N} X$ is (relatively) open, $A_{0} \subset A_{1}$ is compact and plurisubharmonically convex in $\Omega \cap \pi_{N} X, R_{i}: A_{i} \rightarrow(0,1)$ are continuous, $R_{0}<\mu R_{1}\left|A_{0}, R_{1} \leq \gamma\right| A_{1}$. If $A_{1}\left(R_{1}\right)$ is pseudoconvex then $\mathcal{O}(\Omega, E)$ is dense in $\mathcal{O}\left(A_{1}\left(R_{1}\right), E\right)$ over $A_{0}\left[R_{0}\right]$.

Proof. Fix $\alpha_{0}, \alpha_{1}, \alpha_{2}, \alpha_{3} \in \mathcal{A}$ so that $\alpha_{1}<\alpha_{2}<\alpha_{3}, \alpha_{0}<\mu \alpha_{1} / 2$, and choose $\gamma \in \mathcal{A}, \gamma<\alpha=\alpha_{0}$ as in Proposition 4.3b. Further, choose $c$ of (4.2) so that $A_{1} \subset \subset D_{N}\langle\gamma\rangle$. For each $n=0,1, \ldots$ construct a finite $S_{n} \subset D_{n}\left\langle\alpha_{2}\right\rangle$ and a finite open covering $\mathfrak{V}_{n}$ of $D_{n}\left\langle\alpha_{2}\right\rangle$ so that the covering $\mathfrak{V}_{n}\left(\alpha_{2} \mid \pi_{n} X\right)$ of $\Omega_{n}\left\langle\alpha_{2}\right\rangle$ refines the covering

$$
\mathfrak{B}_{n}=\left\{B\left(x, \alpha_{3}(x)\right): x \in S_{n}\right\} .
$$

We arrange that $\mathfrak{B}_{n} \subset \mathfrak{B}_{n+1}$, and set $\mathfrak{B}=\bigcup_{n} \mathfrak{B}_{n}$. The $\mathfrak{U}$-uniform bundle $E$ inherits a $\mathfrak{B}$-uniform structure, and for each $n$ the bundle $E \mid \Omega_{n}\left\langle\alpha_{2}\right\rangle$ inherits a $\mathfrak{B}_{n}-$ and a $\mathfrak{V}_{n}\left(\alpha_{2} \mid \pi_{n} X\right)$-uniform structure. The four uniform structures induce the same topology on $\mathcal{O}(C, E)$ if $C \subset \Omega_{n}\left\langle\alpha_{2}\right\rangle$.

In the first step we apply Corollary 3.5b, with $A_{2}=D_{N}\left\langle\alpha_{1}\right\rangle, A=D_{N}\left\langle\alpha_{2}\right\rangle, r_{0}=$ $R_{0}, r_{1}=R_{1}, r_{2}=\alpha_{1}\left|A_{2}, r=\alpha_{2}\right| A$, to conclude that $\mathcal{O}\left(\Omega_{N}\left\langle\alpha_{1}\right\rangle, E\right)$ is dense in $\mathcal{O}\left(A_{1}\left(R_{1}\right), E\right)$ over $A_{0}\left[R_{0}\right]$.

In the second step we show that for each $n \geq N \mathcal{O}\left(\Omega_{n+1}\left\langle\alpha_{1}\right\rangle, E\right)$ is dense in $\mathcal{O}\left(\Omega_{n}\left\langle\alpha_{1}\right\rangle, E\right)$ over $\bar{\Omega}_{n}\left\langle\alpha_{0}\right\rangle \supset A_{0}\left[R_{0}\right]$. We again apply Corollary $3.5 \mathrm{~b}$, this time with the sets of type (B) (with respect to $\pi_{n+1}$ ) $\bar{\Omega}^{n}\left\langle\alpha_{0}\right\rangle, \Omega^{n}\left\langle\alpha_{1} / 2\right\rangle, \Omega_{n+1}\left\langle\alpha_{1}\right\rangle$, and $\Omega_{n+1}\left\langle\alpha_{2}\right\rangle$ corresponding to $A_{0}\left[r_{0}\right], A_{1}\left(r_{1}\right), A_{2}\left(r_{2}\right)$, resp. $A(r)$ there. That the assumptions of the corollary are satisfied follows from Propositions 4.3, 4.4. We conclude $\mathcal{O}\left(\Omega_{n+1}\left\langle\alpha_{1}\right\rangle, E\right)$ is dense in $\mathcal{O}\left(\Omega^{n}\left\langle\alpha_{1} / 2\right\rangle, E\right)$ over $\bar{\Omega}^{n}\langle\alpha\rangle$. Since

$$
A_{0}\left[R_{0}\right] \subset \bar{\Omega}_{N}\langle\gamma\rangle \subset \bar{\Omega}_{n}\left\langle\alpha_{0}\right\rangle \subset \bar{\Omega}^{n}\left\langle\alpha_{0}\right\rangle \subset \Omega^{n}\left\langle\alpha_{1} / 2\right\rangle \subset \Omega_{n}\left\langle\alpha_{1}\right\rangle
$$

by Propositions $4.3,4.4$, the claim is proved.

Finally, let $e_{B} \in \mathcal{O}(B, \operatorname{Hom}(E, Z))$ denote uniformizing trivializations, $B \in \mathfrak{B}$. Given $f \in \mathcal{O}\left(A_{1}\left(R_{1}\right), E\right)$ and $\varepsilon>0$, successively choose $f_{j} \in \mathcal{O}\left(\Omega_{N+j}\left\langle\alpha_{1}\right\rangle, E\right)$ so that

$$
\begin{array}{cl}
\left\|e_{B} f_{0}-e_{B} f\right\|_{Z}<\varepsilon / 2 & \text { on } B \cap A_{0}\left[R_{0}\right], \text { for all } B \in \mathfrak{B}_{N}, \text { and } \\
\left\|e_{B} f_{j+1}-e_{B} f_{j}\right\|_{Z}<\varepsilon / 2^{j+2} & \text { on } B \cap \bar{\Omega}_{N+j}\left\langle\alpha_{0}\right\rangle, \text { for all } B \in \mathfrak{B}_{N+j},
\end{array}
$$


$j=0,1, \ldots$. In view of Proposition $4.3 \mathrm{c}$ it follows that as $j \rightarrow \infty, e_{B} f_{j}$ converge locally uniformly on $B$, for all $B \in \mathfrak{B}$. The limit will be of form $e_{B} g$ with some $g \in \mathcal{O}(\Omega, E)$ that satisfies $\left\|e_{B} g-e_{B} f\right\|_{Z}<\varepsilon$ on $B \cap A_{0}\left[R_{0}\right]$ for $B \in \mathfrak{B}_{N}$, as needed.

Proposition 4.6. If $\gamma \in \mathcal{A}$ is as in Theorem 4.5, $x_{j} \in \Omega, 0<a_{j} \leq \gamma\left(x_{j}\right)$, and $0<b_{j}<\mu a_{j} / 2$ for $j=0, \ldots, p$, then $\mathcal{O}(\Omega, E)$ is dense in $\mathcal{O}\left(\bigcap_{0}^{p} B\left(x_{j}, a_{j}\right), E\right)$ over $\bigcap_{0}^{p} B\left(x_{j}, b_{j}\right)$.

Proof. Assume $a_{0} \leq a_{1} \leq \ldots$. There is nothing to prove unless $\bigcap_{0}^{p} B\left(x_{j}, a_{j} / 2\right) \neq$ $\emptyset$, so we assume that, too. It follows for each $j$

$$
B\left(x_{j}, a_{j}\right) \supset B\left(x_{0}, a_{0} / 2\right) .
$$

By a translation we can arrange $x_{0}=0$. Apply Theorem 4.5 with $N=0$, to conclude $\mathcal{O}(\Omega, E)$ is dense in $\mathcal{O}\left(B\left(x_{0}, a_{0} / 2\right), E\right)$ over $B\left(x_{0}, b_{0}\right)$. Since

$$
\bigcap_{0}^{p} B\left(x_{j}, a_{j}\right) \supset B\left(x_{0}, a_{0} / 2\right) \quad \text { and } \quad \bigcap_{0}^{p} B\left(x_{j}, b_{j}\right) \subset B\left(x_{0}, b_{0}\right) \text {, }
$$

the proposition follows.

5. The proof of Theorem 1.3. We start with an arbitrary Banach space $(X,\|\|)$ and $\Omega \subset X$ open and connected.

Lemma 5.1. Any locally trivial holomorphic Banach bundle $E \rightarrow \Omega$ can be uniformized.

Proof. Let the fibers of $E$ be isomorphic to the Banach space $Z$. First construct an open covering $\mathfrak{V}$ of $\Omega$ so that $E \mid V$ is trivial for all $V \in \mathfrak{V}$. We can assume each trivialization $e_{V} \in \mathcal{O}(V, \operatorname{Hom}(E, Z))$ extends to a neighborhood of $\bar{V}$, and also that $\mathfrak{V}$ is locally finite. Next for each $x \in \Omega$ choose a neighborhood $W_{x}$ that intersects only finitely many elements of $\mathfrak{V}$. Upon shrinking $W_{x}$ we can arrange that it intersects only those $V_{1} \cap V_{2}, V_{1}, V_{2} \in \mathfrak{V}$, that accumulate at $x$; and then, in view of Corollary 2.5 , that

$$
e_{V_{1}}^{-1} e_{V_{2}} \mid W_{x} \in \mathcal{O}_{u}\left(V_{1} \cap V_{2} \cap W_{x} ; \operatorname{GL}(Z)\right)
$$

for each such $V_{1}, V_{2}$. Thus the trivializations $f_{V \cap W_{x}}=e_{V} \mid W_{x}, V \in \mathfrak{V}, x \in \Omega$, uniformize $E$, as claimed.

Before proving Theorem 1.3, let us quickly review sheaf theoretic notation. Given a collection $\mathfrak{U}=\left\{U_{i}: i \in I\right\}$ of open subsets of $\Omega$ and $p=0,1, \ldots$, we denote by $C^{p}(\mathfrak{U}, E)$ the space of alternating $p$-cochains $f=\left\{f_{s}: s \in I^{p+1}\right\}$, each $f_{s} \in$ $\mathcal{O}\left(\bigcap_{i \in s} U_{i}, E\right)$. We use the convention that $\mathcal{O}(\emptyset, E)=\{0\}$. The coboundary operator is $\delta: C^{p} \rightarrow C^{p+1}$, the space of cocycles is denoted $Z^{p}(\mathfrak{U}, E)$, and the corresponding Čech cohomology group $H^{p}(\mathfrak{U}, E)$. If each $U_{i}$ is pseudoconvex and each $E \mid U_{i}$ trivial, $\mathfrak{U}$ is a Leray covering by Theorem 0.3 of $[\mathrm{Lm} 2]$, whence $H^{p}(\mathfrak{U}, E) \approx H^{p}(\bigcup \mathfrak{U}, E)$.

Proof of Theorem 1.3. We will prove by induction. Given $q \geq 1$, we assume $H^{p}\left(\Omega^{\prime}, E\right)=0$ for all $1 \leq p<q$ and $\Omega^{\prime} \subset \Omega$ pseudoconvex; and we will show $H^{q}(\Omega, E)=0$. We can also assume $\Omega$ connected, hence all fibers of $E$ isomorphic to the same Banach space $\left(Z,\|\|_{Z}\right)$.

By Lemma $5.1 E$ is $\mathfrak{U}$-uniform for some open covering $\mathfrak{U}$ of $\Omega$. Choose $\alpha_{i} \in \mathcal{A}=$ $\mathcal{A}_{\mathfrak{U}}, i=-1, \ldots, 4$, as follows: $\alpha_{4}=\gamma$ as in Theorem $4.5 ; \alpha_{2}<\alpha_{3}<\alpha_{4} ; \alpha_{1}<\alpha_{2}$ is 
such that $B\left(x, \alpha_{1}(x)\right) \subset \Omega_{M}\left\langle\alpha_{2}\right\rangle$ whenever $x \in \bar{\Omega}_{N}\left\langle\alpha_{1}\right\rangle$ and $M \geq N$, cf. Proposition $4.3 \mathrm{~b} ; \alpha_{-1}<\alpha_{0}<\mu \alpha_{1} / 2$. As in the proof of Theorem 4.5, for each $N$ further choose a finite $S_{N} \subset D_{N}\left\langle\alpha_{3}\right\rangle$ and a finite covering $\mathfrak{V}_{N}$ of $D_{N}\left\langle\alpha_{3}\right\rangle$ so that $\mathfrak{V}_{N}\left(\alpha_{3}\right)$ refines the family $\left\{B\left(x, \alpha_{4}(x)\right): x \in S_{N}\right\}$, hence also the family $\mathfrak{U}$. It follows from Corollary $3.5 \mathrm{a}$ that

$$
H^{p}\left(\Omega_{N}\left\langle\alpha_{2}\right\rangle, E\right)=0, \quad \text { for all } N \text { and } p \geq 1 .
$$

Now $B\left(x, \alpha_{4}(x)\right), x \in S_{N}$, cover $\Omega_{N}\left\langle\alpha_{3}\right\rangle$. It can be arranged that more generally, $B\left(x, \alpha_{i}(x)\right), x \in S_{N} \cap D_{N}\left\langle\alpha_{i-1}\right\rangle$, cover $\Omega_{N}\left\langle\alpha_{i-1}\right\rangle, 0 \leq i \leq 4$. It can also be arranged that $S_{N} \subset S_{N+1}$ for all $N$. Let

$$
S=\bigcup_{N} S_{N}, \quad \mathfrak{B}_{i}=\left\{B\left(x, \alpha_{i}(x)\right): x \in S\right\}
$$

and denote by

$$
e_{x} \in \mathcal{O}\left(B\left(x, \alpha_{4}(x)\right), \operatorname{Hom}(E, Z)\right), \quad x \in S,
$$

uniformizing trivializations of $E$. Clearly

$$
\Omega \supset \bigcup \mathfrak{B}_{i} \supset \bigcup_{N} \Omega_{N}\left\langle\alpha_{i-1}\right\rangle=\Omega, \quad 0 \leq i \leq 4 .
$$

By our earlier observation $H^{q}(\Omega, E) \approx H^{q}\left(\mathfrak{B}_{i}, E\right)$, so that it will suffice to show $H^{q}\left(\mathfrak{B}_{4}, E\right)=0$.

Take therefore an $f \in Z^{q}\left(\mathfrak{B}_{4}, E\right)$. (5.1) implies $f \mid \Omega_{N}\left\langle\alpha_{2}\right\rangle=\delta g_{N}$ with some $g_{N} \in C^{q-1}\left(\mathfrak{B}_{4} \mid \Omega_{N}\left\langle\alpha_{2}\right\rangle, E\right)$. We claim $g_{N}$ can be chosen so that their components

$$
g_{N s} \in \mathcal{O}\left(\Omega_{N}\left\langle\alpha_{2}\right\rangle \cap \bigcap_{x \in s} B\left(x, \alpha_{4}(x)\right), E\right), \quad s \in S^{q}
$$

satisfy for all $N$

$$
\left\|e_{x}\left(g_{N+1 s}-g_{N s}\right)\right\|_{Z}<2^{-N}, \quad \text { on } \bigcap_{y \in s} B\left(y, \alpha_{0}(y)\right), x \in s \in\left(S_{N} \cap D_{N}\left\langle\alpha_{1}\right\rangle\right)^{q} .
$$

Indeed, suppose $g_{0}, \ldots, g_{N}$ have already been found, and let the cochain $h \in$ $C^{q-1}\left(\mathfrak{B}_{4} \mid \Omega_{N+1}\left\langle\alpha_{2}\right\rangle, E\right)$ solve $f \mid \Omega_{N+1}\left\langle\alpha_{2}\right\rangle=\delta h$. Thus $\delta\left(h-g_{N}\right)=0$, i.e.,

$$
h-g_{N} \in Z^{q-1}\left(\mathfrak{B}_{4} \mid \Omega_{N}\left\langle\alpha_{2}\right\rangle \cap \Omega_{N+1}\left\langle\alpha_{2}\right\rangle, E\right),
$$

and by the inductive hypothesis $h-g_{N}=\delta k$ with some

$$
k \in C^{q-2}\left(\mathfrak{B}_{4} \mid \Omega_{N}\left\langle\alpha_{2}\right\rangle \cap \Omega_{N+1}\left\langle\alpha_{2}\right\rangle, E\right) .
$$

(When $q=1$ we take this to mean $k$ is a holomorphic section over $\Omega_{N}\left\langle\alpha_{2}\right\rangle \cap \Omega_{N+1}\left\langle\alpha_{2}\right\rangle$, and $\delta k$ means the 0 -cochain $\left(k \mid B\left(x, \alpha_{4}(x)\right)\right)_{x \in S}$.) If $q>1$, apply Proposition 4.6 to the components $k_{t}, t \in\left(S_{N} \cap D_{N}\left\langle\alpha_{1}\right\rangle\right)^{q-1}$ of $k$. Since by the choice of $\alpha_{1}$ these components are holomorphic on $\bigcap_{y \in t} B\left(y, \alpha_{1}(y)\right)$, the proposition gives $l_{t} \in \mathcal{O}(\Omega, E)$ such that

$$
\left\|e_{x}\left(l_{t}-k_{t}\right)\right\|_{Z}<2^{-N} / q \quad \text { on } B\left(x, \alpha_{4}(x)\right) \cap \bigcap_{y \in t} B\left(y, \alpha_{0}(y)\right), \text { if } x \in S_{N} .
$$

For all other $t \in S^{q-1}$ define $l_{t}=0$ and let $l=\left(l_{t}\right) \in C^{q-2}\left(\mathfrak{B}_{0}, E\right)$; then $g_{N+1}=h-\delta l$ satisfies (5.2). If $q=1$ then $k$ is holomorphic on $\Omega_{N}\left\langle\alpha_{1}\right\rangle$, and now Theorem 4.5 gives 
$l \in \mathcal{O}(\Omega, E)$ such that

$$
\left\|e_{x}(l-k)\right\|_{Z}<2^{-N} \quad \text { on } B\left(x, \alpha_{4}(x)\right) \cap \Omega_{N}\left\langle\alpha_{0}\right\rangle \text {, if } x \in S_{N} .
$$

Again, $g_{N+1}=h-\delta l$ will satisfy (5.2).

Now (5.2) implies that for each $s \in S^{q}$, as $N \rightarrow \infty$

$$
g_{N s} \mid \bigcap_{y \in s} B\left(y, \alpha_{0}(y)\right) \rightarrow g_{s} \in \mathcal{O}\left(\bigcap_{y \in s} B\left(y, \alpha_{0}(y)\right), E\right)
$$

clearly $g=\left(g_{s}\right) \in C^{q-1}\left(\mathfrak{B}_{0}, E\right)$ satisfies $\delta g=f \mid \mathfrak{B}_{0}$. Hence the image of $f$ in $H^{q}\left(\mathfrak{B}_{0}, E\right)$ is zero. On the other hand, refinement $\mathfrak{B}_{0} \rightarrow \mathfrak{B}_{4}$ induces an isomorphism in cohomology, which means that the class of $f$ in $H^{q}\left(\mathfrak{B}_{4}, E\right)$ is already zero. Since $f$ was arbitrary, $0=H^{q}\left(\mathfrak{B}_{4}, E\right) \approx H^{q}(\Omega, E)$, q.e.d.

6. The proof of Theorem 1.4. We start by proving a general result on plurisubharmonic domination. Recall the following terminology from [Lm4]. We say that plurisubharmonic domination is possible in a complex manifold $M$ if for any $u: M \rightarrow \mathbb{R}$ that is locally bounded above there is a plurisubharmonic $v: M \rightarrow \mathbb{R}$ such that $u \leq v$. If one can find a Banach space $Z$ and a holomorphic $f: M \rightarrow Z$ so that $u \leq\|f\|_{Z}$, we say in $M$ holomorphic domination is possible.

Lemma 6.1. Let $Y$ be a separable Banach space, $\Omega \subset Y$ open, $P$ a complex manifold, and $\pi: P \rightarrow \Omega$ a holomorphic map. Suppose $u: P \rightarrow \mathbb{R}$ is such that for each $x \in \Omega$ there are a neighborhood $\omega \subset \Omega$ and a plurisubharmonic $v_{\omega}: \pi^{-1} \omega \rightarrow \mathbb{R}$ such that $v_{\omega} \geq u \mid \pi^{-1} \omega$. If in $\Omega$ plurisubharmonic domination is possible then there is a plurisubharmonic $v: P \rightarrow \mathbb{R}$ such that $v \geq u$. If in $\Omega$ holomorphic domination is possible and the $v_{\omega}$ are locally Lipschitz then $v$ can also be chosen locally Lipschitz.

Proof. We shall follow the proof of [Lm4, Theorem 1.4] that constructs seminegative metrics on vector bundles. Cover $\Omega$ by countably many balls of form

$$
\omega_{j}=\left\{x \in X: r_{j}-\left\|x-a_{j}\right\|>0\right\}, \quad j=0,1, \ldots,
$$

so that on $\pi^{-1} \omega_{j}$ plurisubharmonic $v_{j}$ dominate $u$. Define $\chi_{0}(x)=r_{0}-\left\|x-a_{0}\right\|$ and

$$
\chi_{j}(x)=\min \left\{r_{j}-\left\|x-a_{j}\right\|, \frac{1}{j}-r_{i}+\left\|x-a_{i}\right\| ; \quad i<j\right\}, \quad j \geq 1 .
$$

Then the sets $\omega_{j}^{\prime}=\left\{x \in \Omega: \chi_{j}(x)>0\right\} \subset \omega_{j}$ form a locally finite covering of $\Omega$. With $z \in P$ set

$$
\bar{v}(z)=\sup \left\{v_{j}(z)+\log \chi_{j}(\pi z): \omega_{j}^{\prime} \ni \pi z\right\},
$$

which we can also write

$$
\bar{v}=\sup _{j}\left(v_{j}+\pi^{*} \log \chi_{j}\right)
$$

with the understanding that $v_{j}+\pi^{*} \log \chi_{j}$ is extended from $\pi^{-1} \omega_{j}^{\prime}$ to $P$ by $-\infty$. According to [Lm4, Proposition 5.2], valid in an arbitrary separable Banach space, given $x \in \omega_{j}^{\prime}$, there is a Lipschitz continuous, plurisubharmonic $\varphi_{j}: \Omega \rightarrow \mathbb{R}$ such that $\varphi_{j}+\log \chi_{j}$ is plurisubharmonic in a neighborhood of $x$. The sup in (6.1) being locally finite, for each $x \in \Omega$ we find a neighborhood $G \subset \Omega$ and a Lipschitz continuous, 
plurisubharmonic $\varphi_{G}: \Omega \rightarrow \mathbb{R}$ such that

$$
\bar{v}+\pi^{*} \varphi_{G}=\sup _{j}\left\{v_{j}+\pi^{*}\left(\varphi_{G}+\log \chi_{j}\right)\right\} \geq u
$$

is plurisubharmonic in $\pi^{-1} G$ : simply take $\varphi_{G}=\sum_{\omega_{j}^{\prime} \ni x} \varphi_{j}+$ const. Since $\Omega$ is paracompact, and by Lindelöf's theorem, we can assume the sets $G$ form a countable, locally finite covering of $\Omega$. In this situation $[\operatorname{Lm} 4$, Lemma 5.1$]$ provides a $\psi: \Omega \rightarrow \mathbb{R}$ such that $\psi-\varphi_{G}$ is plurisubharmonic in $G$, for all $G$. Moreover, $\psi$ can be chosen to dominate the function $\varphi(x)=\sup _{G \ni x} \varphi_{G}(x)$, which makes $\psi \geq \varphi_{G}$ on $G$. It follows that

$$
v=\bar{v}+\pi^{*} \psi=\left(\bar{v}+\pi^{*} \varphi_{G}\right)+\pi^{*}\left(\psi-\varphi_{G}\right)
$$

is plurisubharmonic and $\geq u$, cf. (6.2).

If in $\Omega$ holomorphic domination is possible and the $v_{j}$ are locally Lipschitz, then [Lm4, Lemma 5.1] provides a locally Lipschitz $\psi$, and $\bar{v}$ is also locally Lipschitz; hence so will be $v$.

Proof of Theorem 1.4. First we show that in $M$ plurisubharmonic domination is possible and $H^{1}(M, E)=0$ for all locally trivial holomorphic Banach bundles $E \rightarrow M$. Let $M$ be biholomorphic to a pseudoconvex open $\Omega$ in a Banach space $Y$. Thus $Y$ is isomorphic to $T_{y} \Omega, y \in \Omega$, which is isomorphic to $T_{x} M, x \in M$, which in turn is complemented in $T_{x} X \approx X$. The upshot is that $Y$ is isomorphic to a complemented subspace of $X$. Let $\iota \in \operatorname{Hom}(Y, X)$ and $\rho \in \operatorname{Hom}(X, Y)$ satisfy $\rho \iota=\mathrm{id}_{Y}$. We shall verify plurisubharmonic domination in $\Omega$. Take a $u: \Omega \rightarrow \mathbb{R}$ that is locally bounded above. Then $\rho^{*} u: \rho^{-1} \Omega \rightarrow \mathbb{R}$ is also such, hence by [Lm4, Theorem 1.6] there is a plurisubharmonic $v \geq \rho^{*} u$ in $\rho^{-1} \Omega$, and $\iota^{*} v$ will then dominate $u$. To show cohomology vanishing, we prove $H^{1}(\Omega, F)=0$ for all locally trivial holomorphic Banach bundles $F \rightarrow \Omega$. Pull back by $\rho$ and $\iota$ induce morphisms

$$
H^{1}(\Omega, F) \stackrel{\rho^{*}}{\rightarrow} H^{1}\left(\rho^{-1} \Omega, \rho^{*} F\right) \stackrel{\iota^{*}}{\rightarrow} H^{1}(\Omega, F)
$$

such that $\iota^{*} \rho^{*}=$ id. Since the group in the middle vanishes by Theorem 1.3, so does $H^{1}(\Omega, F)$.

Second, consider the bundles $\tau=T M, \theta=T X \mid M$, and $\bar{\nu}=\theta / \tau$, all three locally trivial. We claim there is a locally trivial holomorphic Banach bundle $\nu \subset \theta$ such that $\nu \oplus \tau=\theta$. Indeed, the exact sequence $0 \rightarrow \tau \rightarrow \theta \rightarrow \bar{\nu} \rightarrow 0$ induces

$$
0 \rightarrow \operatorname{Hom}(\bar{\nu}, \tau) \rightarrow \operatorname{Hom}(\bar{\nu}, \theta) \rightarrow \operatorname{Hom}(\bar{\nu}, \bar{\nu}) \rightarrow 0,
$$

also exact since $\tau_{x} \subset \theta_{x}$ is complemented for all $x \in M$. In the associated cohomology sequence

$$
H^{0}(M, \operatorname{Hom}(\bar{\nu}, \theta)) \rightarrow H^{0}(M, \operatorname{Hom}(\bar{\nu}, \bar{\nu})) \rightarrow H^{1}(M, \operatorname{Hom}(\bar{\nu}, \tau))=0
$$

there will be an $f \in H^{0}(M, \operatorname{Hom}(\bar{\nu}, \theta))$ that projects on id $\in H^{0}(M, \operatorname{Hom}(\bar{\nu}, \bar{\nu}))$. Thus $f(\bar{\nu})=\nu \subset \theta$ will be complementary to $\tau$. Also $\nu \approx \bar{\nu}$.

Third we construct a holomorphic map $a: \nu \rightarrow X$ and a neighborhood $W \subset \nu$ of the zero section, that we identify with $M$, so that a maps $W$ biholomorphically on an open subset of $X$. Further, $a \mid M=\mathrm{id}_{M}$. Let us introduce the following notation. If $Z$ is a Banach space and $z \in Z$, there is a natural isomorphism between $T_{z} Z$ and $Z$; 
we shall denote by $\tilde{\zeta} \in Z$ the vector corresponding to $\zeta \in T_{z} Z$. Thus $\zeta$ is the velocity vector of the curve $r(t)=z+t \tilde{\zeta}$ at $t=0 \in \mathbb{R}$. We now define

$$
a(\xi)=x+\tilde{\xi}, \quad x \in M, \quad \xi \in \nu_{x} \subset T_{x} X .
$$

One computes that the differential of $a$ at $x \in M$ is an isomorphism; by the implicit function theorem $x$ has an open neighborhood $U \subset \nu$ that $a$ maps biholomorphically on another open neighborhood of $x$. Let $\mathfrak{U}$ be a locally finite covering of $M$ consisting of such neighborhoods $U \subset \nu$. Let $\mathfrak{V}$ be another locally finite covering of $M$ by open sets $V \subset \nu$ such that the closure of each $V$ is contained in some $U \in \mathfrak{U}$. Finally, for each $x \in M$ find an open neighborhood $W_{x} \subset \nu$ so that (1) if $x \in V \in \mathfrak{V}$ then $W_{x}, a\left(W_{x}\right) \subset V$; (2) if $x \in U \in \mathfrak{U}$ then $W_{x} \subset U$; (3) if $a\left(W_{x}\right) \cap V \neq \emptyset, V \in \mathfrak{V}$, then $x \in \bar{V}$. Since sufficiently small neighborhoods of $x$ intersect only finitely many $U$ and $V$, such $W_{x}$ can be found. We claim $a$ is biholomorphic on $W=\bigcup_{x \in M} W_{x}$.

It will suffice to show $a$ is biholomorphic on $W_{x} \cup W_{y}, x, y \in M$. This is obvious if $a\left(W_{x}\right) \cap a\left(W_{y}\right)=\emptyset$. Otherwise let $x \in V \in \mathfrak{V}$; then $W_{x}, a\left(W_{x}\right) \subset V$ by (1), and so $a\left(W_{y}\right) \cap V \neq \emptyset$. Hence $y \in \bar{V}$ by (3). Let $\bar{V} \subset U \in \mathfrak{U}$; (2) implies $W_{y} \subset U$, thus $W_{x}, W_{y} \subset U$. Since $a$ was biholomorphic on $U$, the claim is proved. Also note that $a(W)=\bigcup_{x} a\left(W_{x}\right) \subset X$ is open.

To conclude, recall that a continuous metric on the bundle $\pi: \nu \rightarrow M$ is a continuous $m: \nu \rightarrow[0, \infty)$ that restricts on each fiber $\nu_{x}$ to a norm inducing the topology of the fiber. It is easy to construct a continuous metric $m$ so that $\{\xi \in \nu: m(\xi)<1\}$ is contained in $W$, and its image under $a$ is contained in $O$. By [Lm4, Theorem 1.4] there is a plurisubharmonic metric $p: \nu \rightarrow[0, \infty)$ with $m \leq p$; let $P=\{\xi \in \nu: p(\xi)<1\}$. Thus $a$ maps $P$ biholomorphically on an open $Q \subset O$. All that remains is to show $Q$ is pseudoconvex. For $x \in M$ choose a neighborhood $\omega \subset M$ that is biholomorphic to a pseudoconvex open subset of $Y$; if $\omega$ is small enough then $\nu \mid \omega$ is trivial. It follows that $\pi^{-1} \omega$ is biholomorphic to a pseudoconvex open subset of $X$, and so is $P \cap \pi^{-1} \omega$. Therefore plurisubharmonic domination is possible in $P \cap \pi^{-1} \omega$, and by Lemma 6.1, also in $P$. This, in turn, implies plurisubharmonic domination in $Q$, whence $Q$ is indeed pseudoconvex.

It would be very interesting to investigate what remains true in Theorem 1.4 without the assumption that $M$ is split. We propose the following problem. Let $X$ be a Banach space that one can subject to geometrical conditions, such as existence of a Schauder or unconditional basis. If $O \subset X$ is open and $M \subset O$ a closed complex submanifold, biholomorphic to a pseudoconvex open subset of some Banach space, does it follow that $M$ has a pseudoconvex neighborhood $\subset O$ ? The answer is not known even if $M$ is a pseudoconvex open subset of a linear subspace $Y \subset X$.

\section{REFERENCES}

[A] C.J. Atkin, Boundedness in uniform spaces, topological groups, and homogeneous spaces, Acta Math. Hung., 57 (1991), pp. 213-232.

[C] H. Cartan, Séminaire École Norm. Sup. 4, Fonctions analytiques de plusieurs variables complexes, (1951-52), Paris.

[DG] F. Docquier, H. Grauert, Levisches Problem und Rungescher Satz für Teilgebiete Steinscher Mannigfaltigkeiten, Math. Ann., 140 (1960), pp. 94-123. 
[H] L. Hörmander, An Introduction to Complex Analysis in Several Variables, 3rd ed., North Holland, Amsterdam, 1990.

[J] B. Josefson, Approximations of holomorphic functions in certain Banach spaces, manuscript (2000).

[Li] J. Leiterer, Banach coherent analytic Fréchet sheaves, Math. Nachr., 85 (1978), pp. 91-109. [Lm1] L. Lempert, The Dolbeault complex in infinite dimensions, I, J. Amer. Math. Soc., 11 (1998), pp. $485-520$.

[Lm2] — The Dolbeault complex in infinite dimensions, III, Invent. Math., 142 (2000), pp. 579-603.

[Lm3] - Approximation of holomorphic functions of infinitely many variables, II, Ann. Inst. Fourier Grenoble, 50 (2000), pp. 423-442.

[Lm4] - Plurisubharmonic domination, J. Amer. Math. Soc., to appear.

[M] J. MujicA, Complex Analysis in Banach spaces, North Holland, Amsterdam, 1986.

[N] P. Noverraz, Pseudo-convexité, convexité polynomiale et domaines d'holomorphie en dimension infinie, North Holland, Amsterdam, 1973.

[P1] I. PAtYI, On the Oka principle in a Banach space, I, Math. Ann., 326 (2003), pp. 417-441.

[P2] On the Oka principle in a Banach space, II, Math. Ann., 326 (2003), pp. 443-458.

[P3] - Analytic cohomology of complete intersections in a Banach space, manuscript.

[Sn] I. Singer, Bases in Banach spaces, I-II, Springer, Berlin, 1981.

[Su] Y.T. Siu, Every Stein variety admits a Stein neighborhood, Invent. Math., 38 (1976/77), pp. 89-100. 
L. LEMPERT 